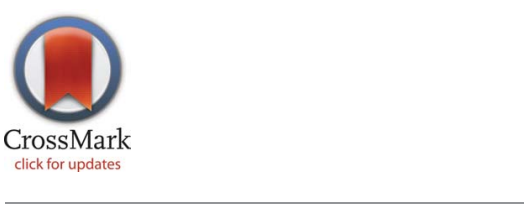

OPEN ACCESS

Citation: Wang L, Peng P, Zhang O, Xu X, Yang S, et al. (2014) High-Dose Statin Pretreatment Decreases Periprocedural Myocardial Infarction and Cardiovascular Events in Patients Undergoing Elective Percutaneous Coronary Intervention: A Meta-Analysis of Twenty-Four Randomized Controlled Trials. PLoS ONE 9(12): e113352. doi:10.1371/journal.pone.0113352

Editor: Luca Testa, Istituto Clinico S. Ambrogio, Italy

Received: May 9, 2014

Accepted: October 22, 2014

Published: December 4, 2014

Copyright: (c) 2014 Wang et al. This is an openaccess article distributed under the terms of the Creative Commons Attribution License, which permits unrestricted use, distribution, and reproduction in any medium, provided the original author and source are credited.

Data Availability: The authors confirm that all data underlying the findings are fully available without restriction. All relevant data are within the paper.

Funding: This work was supported by the National Natural Science Foundation of China NO. 81270285 and NO. 81100198 , the National Construction Program of Key Clinical specialist facility Project and Beijing Municipal Administration of Hospitals Clinical Medicine Development of Special Funding Support code: ZY201303 (http:/l www.bjah.gov.cn/gzdt/kyjy/xzzq/201403/ P020140304488636040596.pdf). The funders had no role in study design, data collection and analysis, decision to publish, or preparation of the manuscript.

Competing Interests: The authors have declared that no competing interests exist.
RESEARCH ARTICLE

\section{High-Dose Statin Pretreatment Decreases Periprocedural Myocardial Infarction and Cardiovascular Events in Patients Undergoing Elective Percutaneous} Coronary Intervention: A Meta-Analysis of Twenty-Four Randomized Controlled Trials

\section{Le Wang, Pingan Peng, Ou Zhang, Xiaohan Xu, Shiwei Yang, Yingxin Zhao, Yujie Zhou*}

Beijing Anzhen Hospital, Capital Medical University, Beijing Institute of Heart Lung and Blood Vessel Disease, The Key Laboratory of Remodeling-Related Cardiovascular Disease, Ministry of Education, Beijing 100029, China

\section{*azzyj12@163.com}

\section{Abstract}

Background: Evidence suggests that high-dose statin pretreatment may reduce the risk of periprocedural myocardial infarction (PMI) and major adverse cardiac events (MACE) for certain patients; however, previous analyses have not considered patients with a history of statin maintenance treatment. In this metaanalysis of randomized controlled trials (RCTs), we reevaluated the efficacy of short-term high-dose statin pretreatment to prevent PMI and MACE in an expanded set of patients undergoing elective percutaneous coronary intervention.

Methods: We searched the PubMed/Medline database for RCTs that compared high-dose statin pretreatment with no statin or low-dose statin pretreatment as a prevention of PMI and MACE. We evaluated the incidence of PMI and MACE, including death, spontaneous myocardial infarction, and target vessel revascularization at the longest follow-up for each study for subgroups stratified by disease classification and prior low-dose statin treatment.

Results: Twenty-four RCTs with a total of 5,526 patients were identified. High-dose statin pretreatment was associated with $59 \%$ relative reduction in $\mathrm{PMI}$ (odds ratio [OR]: $0.41 ; 95 \%$ confidence interval $[\mathrm{Cl}]: 0.34-0.49 ; \mathrm{P}<0.00001)$ and $39 \%$ relative reduction in MACE (OR: $0.61 ; 95 \% \mathrm{Cl}: 0.45-0.83 ; \mathrm{P}=0.002$ ). The benefit of highdose statin pretreatment on MACE was significant for statin-naive patients (OR: $0.69 ; 95 \% \mathrm{Cl}: 0.50-0.95 ; \mathrm{P}=0.02)$ and prior low dose statin-treated patients (OR: 
0.28; 95\% Cl: $0.12-0.65 ; \mathrm{P}=0.003)$; and for patients with acute coronary syndrome (OR: $0.52 ; 95 \% \mathrm{Cl}: 0.34-0.79 ; \mathrm{P}=0.003$ ), but not for patients with stable angina (OR: $0.71 ; 95 \% \mathrm{Cl} 0.45-1.10 ; \mathrm{P}=0.12$ ). Long-term effects on survival were less obvious.

Conclusions: High-dose statin pretreatment can result in a significant reduction in $\mathrm{PMI}$ and MACE for patients undergoing elective PCl. The positive effect of highdose statin pretreatment on PMI and MACE is significant for statin-naïve patients and patients with prior treatment. The positive effect of high-dose statin pretreatment on MACE is significant for patients with acute coronary syndrome.

\section{Introduction}

Percutaneous coronary intervention (PCI) is an important method in the treatment of coronary artery disease. Periprocedural myocardial infarction (PMI), characterized by cardiac biomarker elevation, is a common complication of PCI [1]. Studies have suggested that side branch occlusion, distal embolism, coronary dissection, disruption of collateral flow, and inflammation can result in PMI $[1,2]$. Although most patients who have PMI remain asymptotic and have no change in cardiac function, PMI has been associated with higher mortality [3] . A meta-analysis showed that even small increases in creatine kinase-MB (CK-MB) are associated with significantly higher risk of death during follow-up [4]. Several therapeutic strategies, including statins, antithrombotic agents $[\underline{5}, \underline{6}]$, and $\beta$ blockers $[\underline{7}, \underline{8}]$, have been suggested to reduce PMI. Statins, which inhibit hydroxymethylglutaryl-CoA reductase, have been demonstrated to have pleiotropic effects, including rapid anti-inflammatory and antithrombotic properties $[\underline{9}, \underline{10}]$, antioxidant effects [11], improvement of endothelial dysfunction [12], and stabilization of atherosclerotic plaques [13]. Therefore, statins are regarded as an important agent for the prevention of PMI.

A 2011 meta-analysis [14] comprising 3341 patients from 13 randomized, controlled trials (RCTs) indicated that high-dose statin pretreatment is associated with a significant reduction in PMI and major adverse cardiac events (MACE) in patients undergoing PCI within thirty days. However, more recent clinical trials showed that early use of high-dose statin before PCI did not reduce the occurrence of PMI or improve the long-term clinical outcomes [15-18]. Another more recent meta-analysis of 14 RCTs with 3146 patients [19] showed that highdose statin loading prior to PCI reduced clinical events for patients with acute coronary syndrome (ACS) but not stable angina. However, the latter study was not exhaustive and did not consider subgroups receiving chronic statin therapy prior to the high dose pretreatment. Therefore, a more comprehensive analysis of the benefits of high-dose statin reloading before PCI, including patients under long-term statin therapy, is needed. Thus, we performed a meta-analysis of 24 RCTs comprising 5,526 patients to reevaluate the efficacy of a short-term high- 
dose statin pretreatment to prevent PMI and MACE in patients undergoing elective PCI.

\section{Methods}

\section{Search strategy and inclusion criteria}

In this meta-analysis, two authors independently conducted a thorough search of the PubMed/Medline database for the reports of all RCTs conducted until January 2014 that compare the clinical outcomes of high-dose statin pretreatment with no-statin or low-dose statin pretreatment in patients undergoing elective PCI. Search keywords were "statins", "statin", "atorvastatin", "rosuvastatin", "cervastatin", "simvastatin", "pravastatin", "lovastatin", "fluvastatin", "hydroxymethylglutaryl-CoA", "percutaneous coronary intervention", "PCI”, "stent" and "randomized". We also reviewed the references within related meta-analyses. The primary endpoints of the analysis were the incidences of PMI and MACE. The definition of PMI was taken from the original articles. MACE included death, spontaneous myocardial infarction (MI), and target vessel revascularization (TVR) at the longest follow-up for each study. Studies were included in the present meta-analysis if they met the following criteria: (1) the studies were written in English; (2) the studies were RCTs conducted on humans; (3) the studies did not include patients undergoing primary PCI and non-ST elevation acute coronary syndrome (NSTE-ACS) with high-risk features needing emergency coronary angiography; (4) the studies included a high-dose statin pretreatment therapy group and a no statin or low-dose statin pretreatment therapy group; (5) the definition of PMI in the studies was specified; (6) the incidence of PMI or MACE was specified or could be calculated.

\section{Data extraction and quality assessment}

The data extraction was independently performed by WL and PPA. Any disagreement was resolved by consensus. The risk of bias in each study was evaluated by using the Cochrane Collaboration's instructions [20]. Quality assessment was made on allocation concealment, similarity of baseline characteristics, eligibility criteria, blinding, completeness of follow-up and intention-to-treat analysis. Study quality was quantified using the Jadad score [21].

\section{Statistical analysis}

The statistical analyses were performed using Review Manager 5.2.0. To evaluate the clinical benefit of high-dose statin specifically for patients undergoing elective PCI, subgroup analyses were performed on trials that included only patients with stable angina or patients with ACS, only statin-naive patients, or only patients with prior low-dose statin treatment. The incidence of PMI and MACE was expressed in dichotomous variables, and the results were expressed as odds ratios 
(OR) with 95\% confidence intervals (CI). Heterogeneity across trials was evaluated with $\mathrm{I}^{2}$ statistic, defined as $\mathrm{I}^{2}>50 \%$. If heterogeneity existed a randomeffect model was used; otherwise, a fixed-effect model was chosen. Potential publication bias was assessed with funnel plots. All tests were two-tailed, and a P value of less than 0.05 was regarded as statistically significant.

\section{Results}

\section{Characteristics of studies selected for meta-analysis}

We identified 480 potentially relevant articles from the PubMed/Medline database (Figure 1). After reading the titles and abstracts of all potential articles, the full text of 32 articles was reviewed. By further evaluation, two articles were excluded as duplicate research; and six additional articles were excluded for not meeting our inclusion criteria. Finally, a total of 24 RCTs were examined.

Tables 1, 2 and 3 summarize the main characteristics of the 24 RCTs included in this study. All included studies were published between 2004 and 2013. 5,526 patients were included in the analysis: 2,867 were randomized to a high-dose statin group and 2,659 randomized to receive low-dose or no statin therapy (Control group). Approximately $73 \%$ of enrolled patients were male, $30 \%$ had diabetes, roughly two-thirds of the patients had chronic stable angina, and onethird of the patients presented with ACS. There were five trials $[\underline{18}, \underline{22-25}]$ with

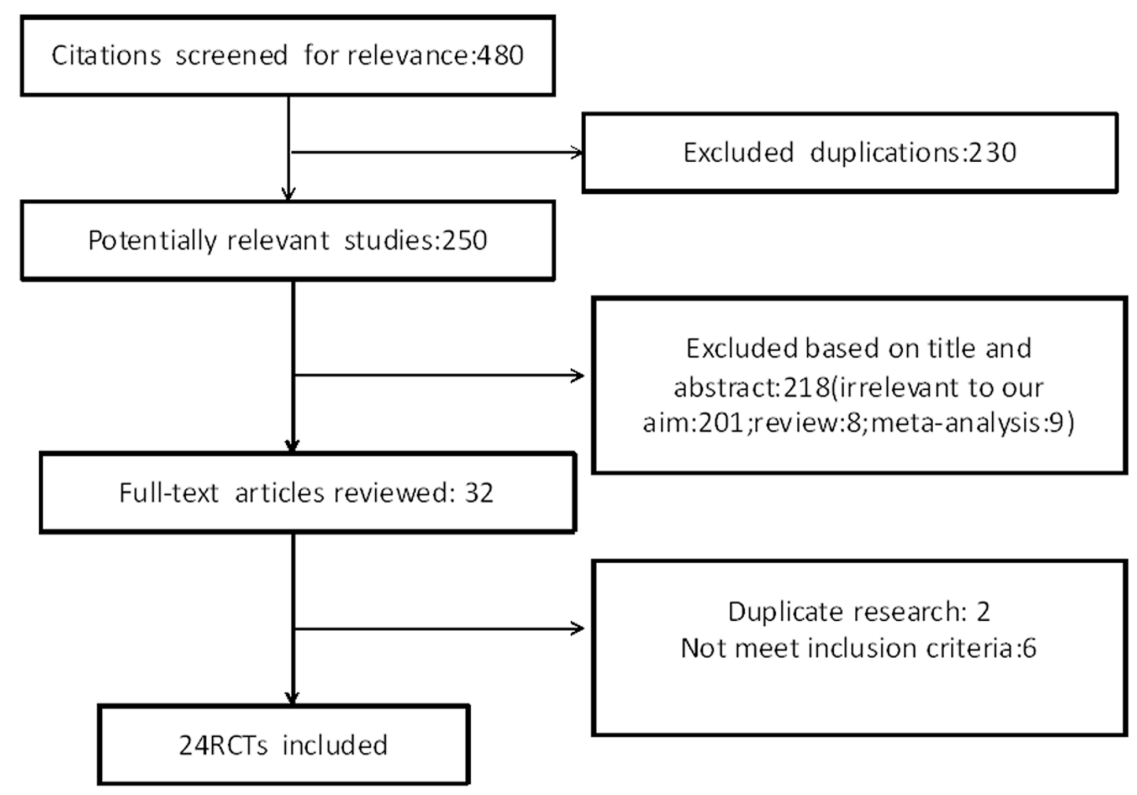

Figure 1. Study selection diagram. 480 potentially relevant publications were identified from Pubmed/ Medline search for key words and research references within related studies. Of these, 230 were duplications and 218 were excluded after further examination of the title and abstract. Of the 32 remaining articles, 2 were from duplicate RCTs and 6 did not meet our inclusion criteria, which left 24 RCTs that were used for our analysis.

doi:10.1371/journal.pone.0113352.g001 
Table 1. Characteristics of included studies.

\begin{tabular}{|c|c|c|c|c|c|c|c|c|}
\hline Trial & patient (n) & $\begin{array}{l}\text { Type of } \\
\text { population }\end{array}$ & $\begin{array}{l}\text { Clinical } \\
\text { presentation }\end{array}$ & Type of statin & $\begin{array}{l}\text { Statin regime } \\
\text { before } \mathrm{PCl}\end{array}$ & $\begin{array}{l}\text { Statin regime } \\
\text { after } \mathrm{PCl}\end{array}$ & Follow up & $\begin{array}{l}\text { PMI } \\
\text { definition }\end{array}$ \\
\hline ARMYDA & 153 & Statin naive & Stable angina & Atorvastatin & $\begin{array}{l}\text { 7-day } 40 \mathrm{mg} / \mathrm{d} \text { before } \\
\mathrm{PCI} V \mathrm{~S} \text { placebo }\end{array}$ & $\begin{array}{l}\text { Atorvastatin } \\
40 \mathrm{mg} / \mathrm{d}\end{array}$ & 30days & $\begin{array}{l}\text { CK- } \\
M B>2 U N L\end{array}$ \\
\hline $\begin{array}{l}\text { Briguori } \\
\text { et al. }\end{array}$ & 451 & Statin naive & $\begin{array}{l}92 \% \text { Stable } \\
\text { angina/asympto- } \\
\text { matic; } 8 \% \\
\text { unstable angina }\end{array}$ & $\begin{array}{l}\text { Atorvastatin29\%;pra- } \\
\text { vastatin,29\%, sim- } \\
\text { vastatin,39\%, fluvsta- } \\
\text { tin, } 3 \%\end{array}$ & $\begin{array}{l}\text { 3-Day pretreatmen- } \\
\text { t(average } 17 \text { days) } \\
\text { VS no statin pretreat- } \\
\text { ment }\end{array}$ & $\begin{array}{l}\text { Same statin as } \\
\text { before } \mathrm{PCl} \text { in the } \\
\text { statin group and } \\
\text { atorvastatin } \\
20 \mathrm{mg} / \mathrm{d} \text { in the } \\
\text { control group }\end{array}$ & In-hospital & $\begin{array}{l}\text { CK- } \\
M B>5 U N L\end{array}$ \\
\hline ARMYDA-ACS & 171 & Statin naive & NSTE-ACS & Atorvastatin & $\begin{array}{l}80 \mathrm{mg} 12 \mathrm{~h} \text { before } \\
\mathrm{PCl}+40 \mathrm{mg} 2 \mathrm{~h} \\
\text { before PCl VS pla- } \\
\text { cebo before PCl }\end{array}$ & $\begin{array}{l}\text { Atorvastatin } \\
40 \mathrm{mg} / \text { day }\end{array}$ & 30days & $\begin{array}{l}\text { CK- } \\
M B>2 U N L\end{array}$ \\
\hline $\begin{array}{l}\text { Bozbas } \\
\text { et al. }\end{array}$ & 93 & Statin naive & Stable angina & Pravastatin & $\begin{array}{l}\text { 1-week pretreatment } \\
\text { with } 10 \mathrm{mg} / \mathrm{d} \text { VS } \\
40 \mathrm{mg} / \mathrm{d} \text { before } \mathrm{PCl} \\
\text { VS no statin pretreat- } \\
\text { ment }\end{array}$ & $\begin{array}{l}\text { Pravastatin } 10 \text { to } \\
40 \mathrm{mg} / \mathrm{d}\end{array}$ & In-hospital & $\begin{array}{l}\text { CK- } \\
M B>3 U N L\end{array}$ \\
\hline $\begin{array}{l}\text { Kinoshita } \\
\text { et al. }\end{array}$ & 42 & Statin naive & Stable angina & Atorvastatin & $\begin{array}{l}5-20 \mathrm{mg} / \mathrm{d} \geq 2 \mathrm{wk} \\
\text { before } \mathrm{PCl} \text { to reach } \\
\text { LDL }<70 \mathrm{mg} / \mathrm{dl} \mathrm{VS} \\
100 \mathrm{mg} / \mathrm{dl}\end{array}$ & $\begin{array}{l}\text { Atorvastatin } 5 \text { to } \\
20 \mathrm{mg} / \mathrm{d}\end{array}$ & 6 months & $\begin{array}{l}\mathrm{CK}- \\
\mathrm{MB}>3 \mathrm{UNL}\end{array}$ \\
\hline NAPLES II & 668 & Statin naive & $\begin{array}{l}98 \% \text { Stable } \\
\text { angina/asympto- } \\
\text { matic; } 2 \% \text { unstable } \\
\text { angina }\end{array}$ & Atorvastatin & $\begin{array}{l}80 \mathrm{mg} \text { within } 24 \mathrm{~h} \\
\text { before PCI VS no } \\
\text { statin pretreatment }\end{array}$ & $\begin{array}{l}\text { atorvastatin } \\
20 \mathrm{mg} / \text { day }\end{array}$ & In-hospital & $\begin{array}{l}\text { CK- } \\
M B>3 U N L\end{array}$ \\
\hline $\begin{array}{l}\text { ARMYDA- } \\
\text { RECAPTURE }\end{array}$ & 383 & Statin-treated & $\begin{array}{l}53 \% \text { stable angina } \\
\text { and } 47 \% \text { NSTE- } \\
\text { ACS }\end{array}$ & Atorvastatin & $\begin{array}{l}80 \mathrm{mg} 12 \mathrm{~h} \text { before } \\
\mathrm{PCl}+40-\mathrm{mg} 2 \mathrm{~h} \\
\text { before PCl VS pla- } \\
\text { cebo }\end{array}$ & $\begin{array}{l}\text { Atorvastatin } \\
40 \mathrm{mg} / \text { day }\end{array}$ & 30days & $\begin{array}{l}\text { CK- } \\
M B>3 U N L\end{array}$ \\
\hline Jia et al. & 228 & Statin naive & $\begin{array}{l}70.6 \% \text { NSTE- } \\
\text { ACS;29.4\%STE- } \\
\text { ACS }\end{array}$ & Simvastatin & $\begin{array}{l}\text { 7-day pretreatment } \\
\text { with } 80 \mathrm{mg} / \mathrm{d} \text { before } \\
\mathrm{PCl} \text { VS } 20 \mathrm{mg} / \mathrm{d} \\
\text { before } \mathrm{PCl}\end{array}$ & $\begin{array}{l}\text { Simvastatin } \\
20 \mathrm{mg} / \mathrm{d}\end{array}$ & In-hospital & $\mathrm{Tnl}>3 \mathrm{UNL}$ \\
\hline Cay et al. & 299 & Statin naive & Stable angina & Rosuvastatin & $\begin{array}{l}\text { Rosuvastatin } 40 \mathrm{mg} \\
24 \mathrm{~h} \text { before } \mathrm{PCI} \text { VS } \\
\text { no statin pretreat- } \\
\text { ment }\end{array}$ & $\begin{array}{l}\text { Rosuvastatin } \\
10-40 \mathrm{mg} / \mathrm{d}\end{array}$ & In-hospital & $\begin{array}{l}\text { CPK- } \\
\mathrm{MB}>3 \mathrm{UNL}\end{array}$ \\
\hline Toso et al. & 161 & Statin naive & Stable angina & Atorvastatin & $\begin{array}{l}80 \mathrm{mg} \text { within } 48 \mathrm{~h} \\
\text { before PCl VS pla- } \\
\text { cebo within } 48 \mathrm{~h} \\
\text { before PCl }\end{array}$ & NA & In-hospital & $\begin{array}{l}\text { CPK- } \\
M B>3 U N L\end{array}$ \\
\hline Yun et al. & 445 & Statin naive & NSTE-ACS & Rosuvastatin & $\begin{array}{l}40 \text { mg within } 7-25 \mathrm{~h} \\
\text { before PCI VS no } \\
\text { statin treatment } \\
\text { before PCl }\end{array}$ & $\begin{array}{l}\text { Rosuvastatin } \\
10 \mathrm{mg} / \text { day }\end{array}$ & 12 months & $\begin{array}{l}\mathrm{CK}- \\
\mathrm{MB}>2 \mathrm{UNL}\end{array}$ \\
\hline $\begin{array}{l}\text { Veselka } \\
\text { et al. }\end{array}$ & 200 & Statin naive & Stable angina & Atorvastatin & $\begin{array}{l}\text { 2-day pretreatment } \\
\text { with atorvastatin } \\
80 \mathrm{mg} / \text { day before } \\
\mathrm{PCI} \text { VS without ator- } \\
\text { vastatin pretreatment }\end{array}$ & $\begin{array}{l}\text { atorvastatin } 20 \\
\text { to } 80 \mathrm{mg} / \text { day }\end{array}$ & 45months & $\mathrm{Tnl}>3 \mathrm{UNL}$ \\
\hline
\end{tabular}

$\mathrm{PCl}=$ percutaneous coronary intervention; $\mathrm{CK}-\mathrm{MB}=$ creatine kinase-MB; UNL= upper normal limit of normal; NSTE-ACS=non-ST-segment elevation acute coronary syndrome;

doi:10.1371/journal.pone.0113352.t001 
Table 2. Characteristics of included studies.

\begin{tabular}{|c|c|c|c|c|c|c|c|c|}
\hline Trial & $\begin{array}{l}\text { Patient } \\
\text { (n) }\end{array}$ & $\begin{array}{l}\text { Type of } \\
\text { population }\end{array}$ & $\begin{array}{l}\text { Clinical } \\
\text { presentation }\end{array}$ & Type of statin & $\begin{array}{l}\text { Statin regime } \\
\text { before } \mathrm{PCI}\end{array}$ & $\begin{array}{l}\text { Statin regime } \\
\text { after } \mathrm{PCl}\end{array}$ & Follow up & $\begin{array}{l}\text { PMI } \\
\text { definition }\end{array}$ \\
\hline Fujii et al. & 80 & Statin naive & Stable angina & Pravastatin & $\begin{array}{l}\text { 4-week pretreat- } \\
\text { ment with } 20 \mathrm{mg} / \mathrm{d} \\
\text { before PCI VS no } \\
\text { pretreatment }\end{array}$ & NA & In-hospital & Tnl $>5 U N L$ \\
\hline Yu et al. & 81 & Statin naive & NSTE-ACS & Atorvastatin & $\begin{array}{l}80 \mathrm{mg} 12 \mathrm{~h} \text { before } \\
\mathrm{PCl}+40 \mathrm{mg} \text { before } \\
\mathrm{PCI} \mathrm{VS} \text { placebo } \\
\text { group }\end{array}$ & $\begin{array}{l}\text { Atorvastatin } \\
20 \mathrm{mg} / \mathrm{d}\end{array}$ & 30days & $\begin{array}{l}\text { CK- } \\
\mathrm{MB}>2 \mathrm{UNL}\end{array}$ \\
\hline Gao et al. & 117 & Statin naive & NSTE-ACS & Rosuvastatin & $\begin{array}{l}20 \mathrm{mg} 12 \mathrm{~h} \text { before } \\
\mathrm{PCl}+10 \mathrm{mg} 2 \mathrm{~h} \\
\text { before } \mathrm{PCI} \text { VS no } \\
\text { treatment }\end{array}$ & $\begin{array}{l}\text { Rosuvastatin } \\
10 \mathrm{mg} / \mathrm{d}\end{array}$ & 6 months & $\begin{array}{l}\text { CK- } \\
\mathrm{MB}>3 \mathrm{UNL}\end{array}$ \\
\hline Jang et al. & 335 & Statin naive & NSTE-ACS & Atorvastatin & $\begin{array}{l}80 \mathrm{mg} 12 \mathrm{~h} \text { and } \\
40 \mathrm{mg} 2 \mathrm{~h} \text { before } \\
\mathrm{PCI} \text { VS no statin } \\
\text { pretreatment }\end{array}$ & $\begin{array}{l}\text { Atorvastatin } \\
40 \mathrm{mg} / \text { day }\end{array}$ & 30days & $\begin{array}{l}\text { CK- } \\
\mathrm{MB}>3 \mathrm{UNL}\end{array}$ \\
\hline $\begin{array}{l}\text { Zemánek } \\
\text { et al. }\end{array}$ & 202 & Statin-treated & Stable angina & Atorvastatin & $\begin{array}{l}\text { 7-day pre-treat- } \\
\text { ment with } 80 \mathrm{mg} / \mathrm{d} \\
\text { VS no statin pre- } \\
\text { treatment }\end{array}$ & NA & In-hospital & $\begin{array}{l}\text { CK- } \\
\mathrm{MB}>3 \mathrm{UNL}\end{array}$ \\
\hline ROMA Trial & 160 & Statin naive & Stable angina & Rosuvastatin & $\begin{array}{l}\text { Rosuvastatin } \\
40 \text { mg within } 24 \mathrm{~h} \\
\text { before PCI VS no } \\
\text { statin }\end{array}$ & $\begin{array}{l}\text { Rosuvastatin } \\
20 \mathrm{mg} / \mathrm{d}\end{array}$ & 12 months & $\begin{array}{l}\text { CK- } \\
\mathrm{MB}>3 \mathrm{UNL}\end{array}$ \\
\hline ROMA II trial & 450 & Statin-treated & Stable angina & $\begin{array}{l}\text { Atorvastatin; } \\
\text { Rosuvastatin }\end{array}$ & $\begin{array}{l}\text { Rosuvastatin } \\
40 \text { mg or } \\
\text { Atorvastatin } \\
80 \mathrm{mg} 24 \mathrm{~h} \text { before } \\
\mathrm{PCI} \text { VS no statin } \\
\text { pretreatment }\end{array}$ & $\begin{array}{l}\text { Rosuvastatin } \\
20 \mathrm{mg} / \mathrm{d} \\
\text { Atorvastatin } \\
40 \mathrm{mg} / \mathrm{d}\end{array}$ & 12 months & $\begin{array}{l}\text { CK- } \\
\mathrm{MB}>3 \mathrm{UNL}\end{array}$ \\
\hline Nafasi et al. & 190 & Statin-treated & $\begin{array}{l}\text { Stable angina } \\
\text { and recent } \mathrm{MI}\end{array}$ & Atorvastatin & $\begin{array}{l}80 \mathrm{mg} \text { within } 24 \mathrm{~h} \\
\text { before } \mathrm{PCl} \mathrm{VS} \\
\text { placebo within } \\
24 \mathrm{~h} \text { before } \mathrm{PCl}\end{array}$ & NA & In-hospital & Tnl $>5 U N L$ \\
\hline Takano et al. & 210 & $\begin{array}{l}\text { statin-treated } \\
\text { and statin- } \\
\text { naive }\end{array}$ & Stable angina & Rosuvastatin & $\begin{array}{l}\text { 5-7day pretreat- } \\
\text { ment with } 20 \mathrm{mg} / \mathrm{d} \\
\text { before PCl VS 5- } \\
7 \text { day pretreatment } \\
\text { with } 2.50 \mathrm{mg} \\
\text { before } \mathrm{PCl}\end{array}$ & $\begin{array}{l}\text { Rosuvastatin } \\
10 \mathrm{mg} / \mathrm{d} \text { AND } \\
\text { rosuvastatin } \\
2.5 \mathrm{mg} / \mathrm{d}\end{array}$ & In-hospital & $\begin{array}{l}\text { CK- } \\
\mathrm{MB}>3 \mathrm{UNL}\end{array}$ \\
\hline Wang et al. & 125 & Statin naive & NSTE-ACS & Rosuvastatin & $\begin{array}{l}20 \mathrm{mg} 2-4 \text { hours } \\
\text { before } \mathrm{PCl} \text { VS } \\
\text { placebo before } \\
\mathrm{PCl}\end{array}$ & $\begin{array}{l}\text { Rosuvastatin } \\
10 \mathrm{mg} / \mathrm{d}\end{array}$ & 30days & $\begin{array}{l}\text { CK- } \\
\mathrm{MB}>3 \mathrm{UNL}\end{array}$ \\
\hline Luo et al. & 67 & Statin naive & NSTE-ACS & Rosuvastatin & $\begin{array}{l}20 \mathrm{mg} 12 \mathrm{~h} \text { before } \\
\mathrm{PCl}+20 \mathrm{mg} 2 \mathrm{~h} \\
\text { before PCI VS no } \\
\text { Statin pretreat- } \\
\text { ment }\end{array}$ & $\begin{array}{l}\text { Rosuvastatin } \\
10 \mathrm{mg} / \mathrm{d}\end{array}$ & 30days & Tnl $>3$ UNL \\
\hline Li et al. & 215 & statin-treated & Stable angina & Atorvastatin & $\begin{array}{l}80 \mathrm{mg} 12 \mathrm{~h} \text { before } \\
\mathrm{PCl} \text { VS } 20 \mathrm{mg} \\
12 \mathrm{~h} \text { before } \mathrm{PCl}\end{array}$ & $\begin{array}{l}\text { Atorvastatin } \\
20 \mathrm{mg} / \mathrm{d}\end{array}$ & 30days & NA \\
\hline
\end{tabular}

$\mathrm{PCl}=$ percutaneous coronary intervention; $\mathrm{CK}-\mathrm{MB}=$ creatine kinase-MB; UNL=upper normal limit of normal; NSTE-ACS=non-ST-segment elevation acute coronary syndrome; ROMA trial=Rosuvastatin pretreatment in patients undergoing elective $\mathrm{PCl}$ to reduce the incidence of periprocedural myocardial necrosis; ROMA II trial=Comparison of high reloading Rosuvastatin and Atorvastatin pretreatment in patients undergoing elective PCI to reduce the incidence of periprocedural myocardial necrosis; $\mathrm{NA}=$ not available.

doi:10.1371/journal.pone.0113352.t002 
Table 3. Clinical and Procedural features in the overall population.

\begin{tabular}{|c|c|c|}
\hline Variables & High-dose statin & Control \\
\hline Population size & 2867 & 2659 \\
\hline Men & 2103(73) & $1921(72)$ \\
\hline Family history of CAD & $536(19)$ & $405(15)$ \\
\hline Hypertension & 1935(67) & $1736(65)$ \\
\hline Diabetes Mellitus & $868(30)$ & $766(29)$ \\
\hline Hyperlipidemia & $701(24)$ & $531(20)$ \\
\hline Smoking & $772(27)$ & $689(26)$ \\
\hline Previous myocardial infarction & $440(15)$ & $455(17)$ \\
\hline Previous $\mathrm{PCl}$ & $219(8)$ & 194(7) \\
\hline Previous CABG & $94(3)$ & $93(3)$ \\
\hline \multicolumn{3}{|l|}{ Clinical presentation } \\
\hline Chronic stable angina & 1950(68) & $1739(66)$ \\
\hline NSTEACS & $886(31)$ & $884(33)$ \\
\hline STEACS & $31(1)$ & $36(1)$ \\
\hline \multicolumn{3}{|l|}{ Medical therapy } \\
\hline$\beta$-blocker & $1318(46)$ & $1318(50)$ \\
\hline ACEI/ARB & $1193(42)$ & $1196(45)$ \\
\hline CCB & $331(12)$ & $326(12)$ \\
\hline \multicolumn{3}{|l|}{ Vessel disease } \\
\hline Multiple vessel & $727(25)$ & $688(26)$ \\
\hline B2/C lesion & $1289(45)$ & $1088(41)$ \\
\hline Thrombus & $40(1.4)$ & $29(1.1)$ \\
\hline \multicolumn{3}{|l|}{ Treated vessel } \\
\hline LM & $14(0.5)$ & $17(0.7)$ \\
\hline LAD & $1250(47)$ & $1178(49)$ \\
\hline LCX & $598(23)$ & $532(22)$ \\
\hline RCA & $778(29)$ & $663(28)$ \\
\hline Sapheonus vein graft & $6(0.2)$ & $11(0.5)$ \\
\hline Multiple vessel PCI & $280(10)$ & $268(10)$ \\
\hline \multicolumn{3}{|l|}{ Antithrombotic therapy } \\
\hline Aspirin & 2674(93) & 2416(91) \\
\hline Clopidogrel & $2460(86)$ & $2188(82)$ \\
\hline Glycoprotein Ilb/IIla inhibitors & $295(10)$ & $315(12)$ \\
\hline
\end{tabular}

$\mathrm{CAD}=$ coronary artery disease $\mathrm{PCl}=$ percutaneous coronary syndrome; $\mathrm{CABG}=$ coronary artery bypass graft; NSTEACS = non-ST segment elevation acute coronary syndrome; STEACS =ST segment elevation acute coronary syndrome; $\mathrm{ACEI}=$ angiotensin converting enzyme inhibitor; $\mathrm{ARB}=\mathrm{Angiotensin} \mathrm{II}$ receptor antagonist; $\mathrm{CCB}=$ calcium channel blocker; $\mathrm{LM}=$ left main; $\mathrm{LAD}=$ left anterior descending; $\mathrm{LCX}=$ left circumflex; $\mathrm{RCA}=$ right coronary artery.

doi:10.1371/journal.pone.0113352.t003

only prior low dose statin-treated patients, eighteen $[\underline{16}, \underline{17}, \underline{26}-\underline{41}]$ with only statin-näve patients, and one [42] with both prior low-dose statin-treated and statin-nä̈ve patients. Twelve studies $[\underline{16}, \underline{18}, \underline{22}, \underline{24}, \underline{26}, \underline{29}, \underline{30}, \underline{33}, \underline{34}, \underline{36}, \underline{39}, \underline{42}]$ included patients with stable angina, seven studies $[17,28,35,37,38,40,41]$ included patients with ACS, and five studies $[23,25,27,31,32]$ included patients with mixed presentations. Fourteen trials $[\underline{16}-18, \underline{22-28}, \underline{30}, \underline{31}, \underline{34}, \underline{37}]$ used 
atorvastatin, eight $[\underline{24}, \underline{33}, \underline{35}, \underline{38}-\underline{42}]$ used rosuvastatin, three $[\underline{27}, 29, \underline{36}]$ used pravastatin, one [27] used fluvastatin, and two [27, 32] used simvastatin. The duration of statin pretreatment ranged from two hours to four weeks. All patients in these trials received statin therapy after PCI, irrespective of the initial assignment. Eighteen studies [17, 18, 22, 23, 25-29, 31-34, 36, 37, 40-42] included short-term follow-up (in-hospital or up to 30 days), and six studies $[\underline{16}, \underline{24}, \underline{30}, \underline{35}, \underline{38}, \underline{39}]$ had follow-up duration between six months and forty-five months. The definition of PMI varied among the included studies as follows: Four studies $[\underline{26}, \underline{28}, \underline{35}, \underline{37}]$ demanded a CK-MB concentration greater than two times above the upper normal limit (ULN), thirteen studies $[\underline{17}, \underline{18}, \underline{23}, \underline{24}, \underline{29}$ $31,33,34,38,39,41,42]$ a CK-MB concentration greater than three times above ULN, and one study [27] a CK-MB concentration greater than five times above ULN. Other studies employed the Troponin I (TnI) concentration to define PMI. Three studies $[\underline{16}, \underline{32}, \underline{40}]$ required a $\mathrm{TnI}$ concentration greater than three times above ULN and two studies $[\underline{25}, 26]$ a TnI concentration greater than five times above ULN. Table 4 shows the quality characteristics of the included RCTs. Seventy-nine percent of the trials are high-quality trials, according to the Jadad score. Three trials $[\underline{23}, \underline{25}, \underline{28}]$ used allocation concealment and blinding.

\section{Effect of statin pretreatment on clinical outcome}

Outcome data for PMI were available from 23 RCTs, but not from the study by Li Q et al. [22]. The overall outcomes, based on the fixed effects model, showed that high-dose statin pretreatment was associated with a 59\% relative reduction in PMI $\left(\mathrm{OR}=0.41,95 \%\right.$ CI $\left.0.34-0.49, \mathrm{P}<0.00001 ; \mathrm{I}^{2}=20 \%\right)$ (Figure 2). The relative symmetry in the funnel plot shows that there was no evidence to suggest any publication bias (Figure 3).

Outcome data for MACE were available from 16 RCTs, whereas the other 8 RCTs (by Bozbas et al. [29], Jia et al. [32], Cay et al. [33], Toso et al. [34], Fujii et al. [36], Zemanek et al. [18], Nafasi et al. [25], and Takano et al. [42]) only collected data about PMI. The overall outcomes, based on the fixed effects model, showed that high-dose statin pretreatment was associated with a $39 \%$ relative reduction in MACE $\left(\mathrm{OR}=0.61,95 \%\right.$ CI $\left.0.45-0.83, \mathrm{P}=0.002 ; \mathrm{I}^{2}=31 \%\right)$ (Figure 4). There was no evidence of significant heterogeneity among the 16 trials $(\mathrm{P}=0.13$; $\mathrm{I}^{2}=31 \%$ ), and the funnel plot analysis did not suggest the presence of any publication bias (Figure 5). These data confirm the reliability of this set of data and also verify the overall beneficial effects of high-dose statin loading on PMI and MACE using a greatly expanded data set relative to previous meta-analyses $[14,19]$.

\section{Analysis of subgroups with different types of disease presentation}

To determine whether the outcome of high-dose statin pretreatment prior to PCI differs for patients with stable angina, ACS, or a mixed disease presentation, we assessed subgroups of patients according to their disease classification. High-dose 
Table 4. Quality of included RCTs.

\begin{tabular}{|c|c|c|c|c|c|c|c|c|c|}
\hline \multirow[t]{2}{*}{ Author, Year } & \multirow[t]{2}{*}{$\begin{array}{l}\text { Jadad } \\
\text { score }\end{array}$} & \multirow[t]{2}{*}{$\begin{array}{l}\text { Allocation } \\
\text { Concealment }\end{array}$} & \multirow[t]{2}{*}{$\begin{array}{l}\text { Similarity of } \\
\text { baseline } \\
\text { characteristics }\end{array}$} & \multirow[t]{2}{*}{$\begin{array}{l}\text { Eligibility } \\
\text { Criteria }\end{array}$} & \multicolumn{3}{|l|}{ Blinding } & \multirow[t]{2}{*}{$\begin{array}{l}\text { Completeness } \\
\text { of Follow-up }\end{array}$} & \multirow[t]{2}{*}{$\begin{array}{l}\text { Intention- } \\
\text { to-treat }\end{array}$} \\
\hline & & & & & $\begin{array}{l}\text { Outcome } \\
\text { Assessor }\end{array}$ & $\begin{array}{l}\text { Care } \\
\text { provider }\end{array}$ & Patient & & \\
\hline ARMYDA,2004 & 5 & NA & Yes & Yes & No & Yes & Yes & Yes & Yes \\
\hline Briguori,2004 & 4 & Yes & Yes & Yes & No & No & No & Yes & Yes \\
\hline ARMYDA-ACS 2007 & 6 & Yes & Yes & Yes & No & Yes & Yes & Yes & Yes \\
\hline Kinoshita,2007 & 4 & NA & Yes & Yes & No & No & NA & Yes & Yes \\
\hline Bozbas,2007 & 4 & NA & Yes & Yes & No & No & Yes & Yes & Yes \\
\hline Naples II,2009 & 5 & Yes & Yes & Yes & No & No & No & Yes & Yes \\
\hline $\begin{array}{l}\text { ARMYDA- } \\
\text { RECAPTURE,2009 }\end{array}$ & 6 & Yes & Yes & Yes & No & Yes & Yes & Yes & Yes \\
\hline Jia,2009 & 4 & NA & Yes & Yes & Yes & No & No & Yes & Yes \\
\hline Cay,2010 & 3 & NA & Yes & Yes & No & No & No & Yes & Yes \\
\hline Toso,2011 & 3 & No & Yes & Yes & No & No & No & Yes & Yes \\
\hline Yun,2011 & 4 & NA & Yes & Yes & No & No & No & Yes & Yes \\
\hline Veselka,2011 & 3 & NA & Yes & Yes & No & No & No & Yes & Yes \\
\hline Fujii,2011 & 4 & NA & Yes & Yes & No & No & No & Yes & Yes \\
\hline Yu,2011 & 4 & NA & Yes & Yes & No & Yes & Yes & Yes & Yes \\
\hline Gao,2012 & 3 & NA & Yes & Yes & No & No & No & Yes & Yes \\
\hline Jang,2013 & 4 & NA & Yes & Yes & No & No & Yes & Yes & Yes \\
\hline Zemánek,2013 & 4 & NA & Yes & Yes & No & No & Yes & Yes & Yes \\
\hline ROMA II,2013 & 5 & Yes & Yes & Yes & No & No & No & Yes & Yes \\
\hline Nafasi,2013 & 6 & Yes & Yes & Yes & Yes & Yes & Yes & Yes & Yes \\
\hline ROMA,2013 & 4 & Yes & Yes & Yes & No & No & No & Yes & Yes \\
\hline Takano,2013 & 3 & No & Yes & Yes & No & No & No & Yes & Yes \\
\hline Wang,2013 & 4 & NA & Yes & Yes & Yes & Yes & Yes & Yes & Yes \\
\hline Luo,2013 & 4 & NA & Yes & Yes & NA & NA & NA & Yes & Yes \\
\hline Li,2013 & 4 & NA & Yes & Yes & NA & NA & NA & Yes & Yes \\
\hline
\end{tabular}

$\mathrm{NA}=$ not available

doi:10.1371/journal.pone.0113352.t004

statin pretreatment was associated with $63 \%$ relative reduction in PMI for patients with stable angina $\left(\mathrm{OR}=0.37,95 \%\right.$ CI $\left.0.28-0.49, \mathrm{P}<0.00001 ; \mathrm{I}^{2}=44 \%\right) ; 61 \%$ relative reduction in PMI for patients with ACS ( $\mathrm{OR}=0.39,95 \%$ CI 0.26-0.59, $\left.\mathrm{P}<0.00001 ; \mathrm{I}^{2}=10 \%\right)$; and $53 \%$ relative reduction in PMI for patients with mixed presentation $\left(\mathrm{OR}=0.47,95 \%\right.$ CI $0.34-0.65, \mathrm{P}<0.00001 ; \mathrm{I}^{2}=0 \%$ ) (Figure 2).

We also assessed the stable angina, ACS, and mixed disease subgroups for their effect on MACE. For the subgroup with ACS, the incidence of MACE was significantly lower in the high-dose statin group than in the control group $\left(\mathrm{OR}=0.52\right.$, 95\% CI $\left.0.34-0.79, \mathrm{P}=0.003 ; \mathrm{I}^{2}=38 \%\right)$; however, for patients with stable angina or mixed presentation, there was no significant difference in MACE between the high-dose statin group and the control group $(\mathrm{OR}=0.71,95 \% \mathrm{CI}$ $0.45-1.10, \mathrm{P}=0.12 ; \mathrm{I}^{2}=41 \%$; and $\mathrm{OR}=1.48,95 \%$ CI $0.25-8.90, \mathrm{P}=0.67 ; \mathrm{I}^{2}=31 \%$, 


\begin{tabular}{|c|c|c|c|c|c|c|c|c|}
\hline Studv or Subgroup & \multicolumn{2}{|c|}{ High-dose statin } & \multicolumn{2}{|c|}{ Control } & Weight & $\begin{array}{l}\text { Odds Ratio } \\
\text { M-H, Fixed, } 95 \% \mathrm{Cl}\end{array}$ & \multicolumn{2}{|c|}{$\begin{array}{l}\text { Odds Ratio } \\
\text { M-H, Fixed, } 95 \% \mathrm{Cl}\end{array}$} \\
\hline \multicolumn{9}{|l|}{ 1.1.1 Stable } \\
\hline ARMYDA2004 & 4 & 76 & 14 & 77 & $3.7 \%$ & $0.25[0.08,0.80]$ & & \\
\hline Bozbas2007 & 0 & 29 & 1 & 64 & $0.3 \%$ & $0.72[0.03,18.14]$ & & \\
\hline Cay 2010 & 1 & 153 & 16 & 146 & $4.5 \%$ & $0.05[0.01,0.41]$ & & \\
\hline Fujii2011 & 5 & 40 & 13 & 40 & $3.2 \%$ & $0.30[0.09,0.93]$ & & \\
\hline Kinoshita2007 & 2 & 21 & 4 & 21 & $1.0 \%$ & $0.45[0.07,2.76]$ & & \\
\hline ROMA II2013 & 30 & 350 & 29 & 100 & $11.5 \%$ & $0.23[0.13,0.41]$ & & \\
\hline ROMA2013 & 7 & 80 & 21 & 80 & $5.3 \%$ & $0.27[0.11,0.68]$ & & \\
\hline Takano2013 & 9 & 104 & 20 & 106 & $5.0 \%$ & $0.41[0.18,0.94]$ & & \\
\hline Toso2011 & 8 & 77 & 19 & 84 & $4.5 \%$ & $0.40[0.16,0.97]$ & & \\
\hline Veselka2011 & 10 & 100 & 12 & 100 & $3.0 \%$ & $0.81[0.33,1.98]$ & & - \\
\hline Zemánek2013 & 15 & 100 & 14 & 102 & $3.3 \%$ & $1.11[0.50,2.44]$ & & \\
\hline Subtotal $(95 \% \mathrm{Cl})$ & & 1130 & & 920 & $45.4 \%$ & $0.37[0.28,0.49]$ & & \\
\hline Total events & 91 & & 163 & & & & & \\
\hline \multicolumn{9}{|c|}{$\begin{array}{l}\text { Heterogeneity: } \mathrm{Chi}^{2}=17.98, \mathrm{df}=10(\mathrm{P}=0.06) ; \mathrm{I}^{2}=44 \% \\
\text { Test for overall effect: } Z=6.88(\mathrm{P}<0.00001)\end{array}$} \\
\hline \multicolumn{9}{|l|}{ 1.1.2 ACS } \\
\hline ARMYDA-ACS2007 & 4 & 86 & 13 & 85 & $3.5 \%$ & $0.27[0.08,0.87]$ & & \\
\hline Gao2012 & 0 & 59 & 4 & 58 & $1.3 \%$ & $0.10[0.01,1.93]$ & & \\
\hline Jang 2013 & 12 & 163 & 16 & 172 & $4.0 \%$ & $0.77[0.35,1.69]$ & & - \\
\hline Luo2013 & 3 & 31 & 11 & 36 & $2.6 \%$ & $0.24[0.06,0.97]$ & & \\
\hline Wang2013 & 5 & 62 & 14 & 63 & $3.6 \%$ & $0.31[0.10,0.91]$ & & \\
\hline Yu2011 & 1 & 41 & 8 & 40 & $2.2 \%$ & $0.10[0.01,0.84]$ & & \\
\hline Yun2011 & 13 & 225 & 25 & 220 & $6.6 \%$ & $0.48[0.24,0.96]$ & & \\
\hline Subtotal $(95 \% \mathrm{Cl})$ & & 667 & & 674 & $23.7 \%$ & $0.39[0.26,0.58]$ & & \\
\hline Total events & 38 & & 91 & & & & & \\
\hline \multirow{2}{*}{\multicolumn{9}{|c|}{$\begin{array}{l}\text { Heterogeneity: } \mathrm{Chi}^{2}=6.66 \text {, df }=6(\mathrm{P}=0.35) ;\left.\right|^{2}=10 \% \\
\text { Test for overall effect: } Z=4.67(P<0.00001)\end{array}$}} \\
\hline & & & & & & & & \\
\hline \multicolumn{9}{|l|}{ 1.1.3 Mixed presentation } \\
\hline ARMYDA-RECAPTURE2009 & 7 & 192 & 17 & 191 & $4.6 \%$ & $0.39[0.16,0.96]$ & & \\
\hline Briguori2004 & 18 & 226 & 35 & 225 & $9.0 \%$ & $0.47[0.26,0.86]$ & & \\
\hline Jia2009 & 1 & 113 & 5 & 115 & $1.4 \%$ & $0.20[0.02,1.71]$ & & \\
\hline Nafasi2013 & 3 & 95 & 10 & 95 & $2.7 \%$ & $0.28[0.07,1.04]$ & & \\
\hline Naples II 2009 & 32 & 338 & 52 & 330 & $13.3 \%$ & $0.56[0.35,0.89]$ & & \\
\hline Subtotal $(95 \% \mathrm{CI})$ & & 964 & & 956 & $30.9 \%$ & $0.47[0.34,0.65]$ & & \\
\hline Total events & 61 & & 119 & & & & & \\
\hline \multicolumn{9}{|c|}{$\begin{array}{l}\text { Heterogeneity: } \mathrm{Chi}^{2}=1.94, \mathrm{df}=4(\mathrm{P}=0.75) ;\left.\right|^{2}=0 \% \\
\text { Test for overall effect: } Z=4.58(P<0.00001)\end{array}$} \\
\hline Total $(95 \% \mathrm{Cl})$ & & 2761 & & 2550 & $100.0 \%$ & $0.41[0.34,0.49]$ & $\bullet$ & \\
\hline Total events & 190 & & 373 & & & & & \\
\hline \multirow{2}{*}{\multicolumn{7}{|c|}{$\begin{array}{l}\text { Heterogeneity: } \mathrm{Chi}^{2}=27.50, \mathrm{df}=22(\mathrm{P}=0.19) ; \mathrm{I}^{2}=20 \% \\
\text { Test for overall effect: } Z=9.43(\mathrm{P}<0.00001)\end{array}$}} & $0.01 \quad 0.1$ & $\begin{array}{lll}1 & 10 & 100\end{array}$ \\
\hline & & & & & & & ours[High-dose statin] & Favours [Control] \\
\hline
\end{tabular}

Figure 2. Forest plots for PMI incidence for patients stratified by different clinical presentation. Forest plots were generated using Review Manager 5.2.0 for patients with stable angina, ACS or mixed disease presentation from individual and pooled trials. The incidence of PMI was expressed as a dichotomous variable, and the results were expressed as odds ratios (OR) with $95 \%$ confidence intervals (Cl). Heterogeneity across trials was evaluated with $\mathrm{I}^{2}$ statistic, defined as $\mathrm{I}^{2}>50 \%$. Because the heterogeneity was low, a fixed-effect model was used.

doi:10.1371/journal.pone.0113352.g002

respectively) (Figure 4). These findings confirm and extend previous findings that suggest that high dose statin loading is only effective for ACS patients on MACE [19]. 


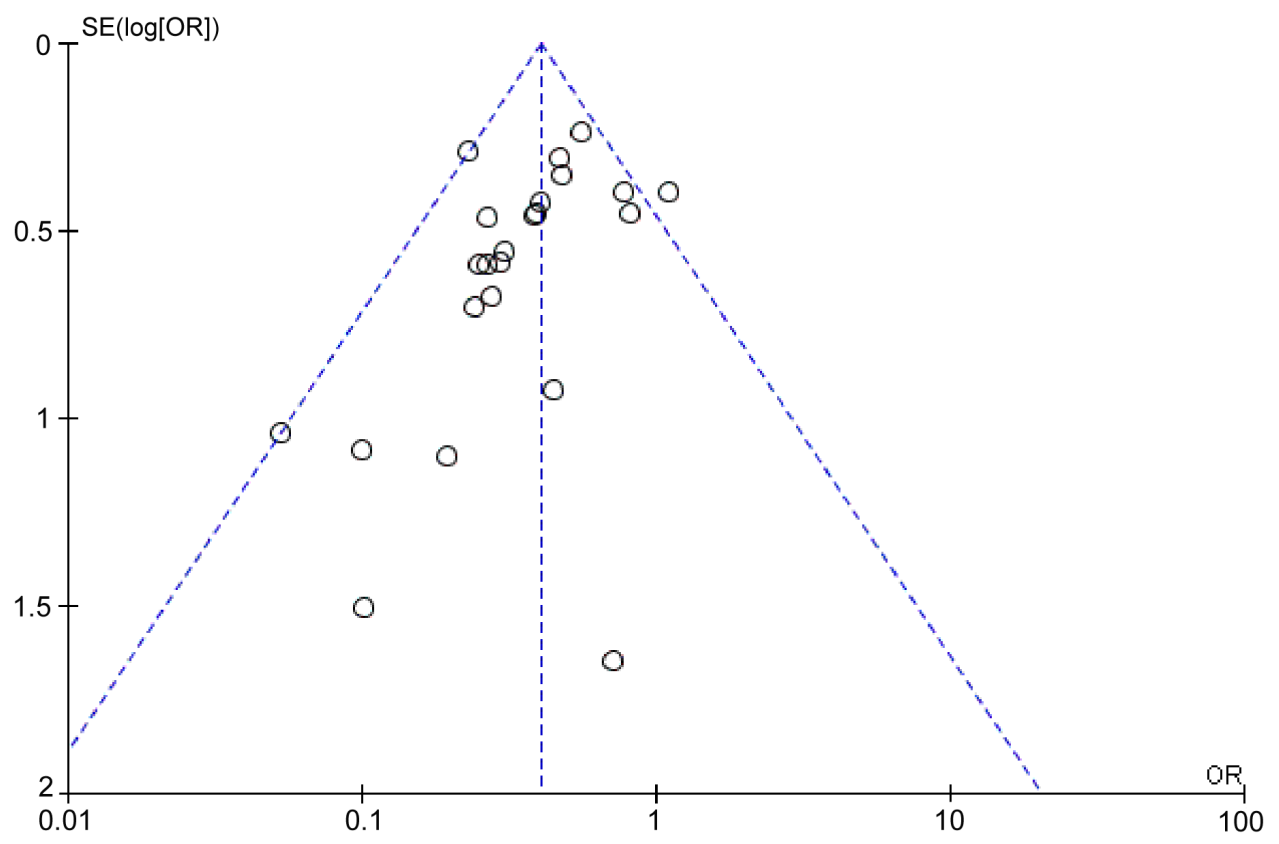

Figure 3. Funnel plot for PMI incidence to rule out publication bias. Funnel plot was generated using a fixed-effect model by Review Manager 5.2.0.

doi:10.1371/journal.pone.0113352.g003

\section{Analysis of subgroups according to prior low-dose statin use}

To determine whether chronic low-dose statin therapy prior to the high-dose PCI pretreatment affects clinical outcome, we grouped the patients according to whether or not they had a history of low-dose statin treatment prior to the highdose pre-PCI treatment. For the subgroup of statin-naive patients, high-dose statin pretreatment was associated with a lower incidence of $\mathrm{PMI}(\mathrm{OR}=0.41,95 \%$ CI $0.33-0.50, \mathrm{P}<0.00001 ; \mathrm{I}^{2}=0 \%$ ) (Figure 6). For the subgroup with prior lowdose statin treatment, heterogeneity was found across trials $\left(\mathrm{I}^{2}=63 \%\right)$, and a randomized model was therefore used. Similar to the results for the statin-naive patients, the incidence of PMI for the prior low-dose statin patients was lower in the high-dose statin group than that in the control group $(\mathrm{OR}=0.44,95 \% \mathrm{CI}$ $0.23-0.85, \mathrm{P}=0.01$ ) (Figure 7). Furthermore, assessment of the effects on MACE suggested that high-dose statin loading is beneficial for both statin-naïe patients $\left(\mathrm{OR}=0.69,95 \%\right.$ CI $\left.0.50-0.95, \mathrm{P}=0.02 ; \mathrm{I}^{2}=34 \%\right)$ and prior low-dose statin patients $\left(\mathrm{OR}=0.28,95 \%\right.$ CI $\left.0.12-0.65, \mathrm{P}=0.003 ; \mathrm{I}^{2}=0 \%\right)$ (Figure 8$)$. These data provide evidence using meta-analysis or multiple RCTs to support the use of high-dose statin loading prior to PCI for patients having prior low-statin chronic therapeutic use. 


\begin{tabular}{|c|c|c|c|c|c|c|c|c|c|}
\hline \multirow[b]{2}{*}{ Studv or Subgroup } & \multicolumn{2}{|c|}{ High-dose statin } & \multicolumn{2}{|c|}{ Control } & \multirow[b]{2}{*}{ Weight } & \multirow{2}{*}{$\begin{array}{c}\text { Odds Ratio } \\
\text { M-H.Fixed, } 95 \% \mathrm{Cl}\end{array}$} & \multirow{2}{*}{\multicolumn{3}{|c|}{$\begin{array}{c}\text { Odds Ratio } \\
\text { M-H, Fixed, } 95 \% \mathrm{Cl}\end{array}$}} \\
\hline & Events & Total & Events & Total & & & & & \\
\hline \multicolumn{10}{|l|}{ 3.1.1 Stable } \\
\hline ARMYDA2004 & 0 & 76 & 0 & 77 & & Not estimable & & & \\
\hline Kinoshita2007 & 0 & 20 & 1 & 21 & $1.3 \%$ & $0.33[0.01,8.67]$ & & & \\
\hline Li2013 & 0 & 106 & 2 & 109 & $2.3 \%$ & $0.20[0.01,4.25]$ & & & \\
\hline ROMA $\| 2013$ & 11 & 350 & 10 & 100 & $14.0 \%$ & $0.29[0.12,0.71]$ & & & \\
\hline ROMA2013 & 4 & 80 & 6 & 80 & $5.3 \%$ & $0.65[0.18,2.39]$ & & & \\
\hline Veselka2011 & 35 & 100 & 33 & 100 & $19.9 \%$ & $1.09[0.61,1.96]$ & & & \\
\hline Subtotal $(95 \% \mathrm{CI})$ & & 732 & & 487 & $427 \%$ & $0.71[0.45,1.10]$ & & & \\
\hline Total events & 50 & & 52 & & & & & & \\
\hline \multicolumn{10}{|c|}{ Heterogeneity: $\mathrm{Chi}^{2}=6.81, \mathrm{df}=4(\mathrm{P}=0.15) ; \mathrm{I}^{2}=41 \%$} \\
\hline \multicolumn{10}{|c|}{ Test for overall effect: $Z=1.54(P=0.12)$} \\
\hline \multicolumn{10}{|l|}{ 3.1.2 ACS } \\
\hline ARMYDA-ACS2007 & 0 & 86 & 1 & 85 & $1.4 \%$ & $0.33[0.01,8.11]$ & & & \\
\hline Gao2012 & 2 & 59 & 10 & 58 & $9.0 \%$ & $0.17[0.04,0.81]$ & & & \\
\hline Jang2013 & 13 & 163 & 10 & 172 & $8.3 \%$ & $1.40[0.60,3.30]$ & & & \\
\hline Lu02013 & 1 & 31 & 2 & 36 & $1.7 \%$ & $0.57[0.05,6.57]$ & & & \\
\hline Wang2013 & 0 & 62 & 0 & 63 & & Not estimable & & & \\
\hline YU2011 & 0 & 41 & 1 & 40 & $1.4 \%$ & $0.32[0.01,8.02]$ & & & \\
\hline Yun2011 & 18 & 225 & 39 & 220 & $33.6 \%$ & $0.40[0.22,0.73]$ & & & \\
\hline Subtotal $(95 \% \mathrm{CI})$ & & 667 & & 674 & $55.4 \%$ & $0.52[0.34,0.79]$ & & & \\
\hline Total events & 34 & & 63 & & & & & & \\
\hline \multicolumn{10}{|c|}{ Heterogeneity: $\mathrm{Chi}^{2}=8.08, \mathrm{df}=5(\mathrm{P}=0.15) ; \mathrm{I}^{2}=38 \%$} \\
\hline \multicolumn{10}{|c|}{ Test for overall effect: $Z=3.01(P=0.003)$} \\
\hline \multicolumn{10}{|l|}{ 3.1.3 Mixed presentation } \\
\hline ARMYDA-RECAPTURE 2009 & 0 & 192 & 1 & 191 & $1.4 \%$ & $0.33[0.01,8.15]$ & & & \\
\hline Briguori2004 & 0 & 226 & 0 & 225 & & Not estimable & & & \\
\hline Naples II 2009 & 2 & 338 & 0 & 330 & $0.5 \%$ & $4.91[0.23,102.68]$ & & & \\
\hline Subtotal $(95 \% \mathrm{CI})$ & & 756 & & 746 & $1.9 \%$ & $1.48[0.25,8.90]$ & & & \\
\hline Total events & 2 & & 1 & & & & & & \\
\hline \multicolumn{10}{|c|}{ Heterogeneity: $\mathrm{Chi}^{2}=1.44, \mathrm{df}=1(\mathrm{P}=0.23) ;\left.\right|^{2}=31 \%$} \\
\hline \multicolumn{10}{|c|}{ Test for overall effect: $Z=0.43(P=0.67)$} \\
\hline Total $(95 \% \mathrm{Cl})$ & & 2155 & & 1907 & $100.0 \%$ & $0.61[0.45,0.83]$ & 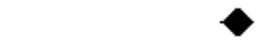 & & \\
\hline Total events & 86 & & 116 & & & & & & \\
\hline $\begin{array}{l}\text { Heterogeneity: } \text { Chi }^{2}=17.48, \\
\text { Test for overall effect: } Z=3.1\end{array}$ & $\begin{array}{l}=12(P=0 \\
P=0.002)\end{array}$ & $i^{2}=319$ & & & & Favo & $\begin{array}{ll}0.01 & 0.1 \\
\text { ours[High-dose statin] }\end{array}$ & $\begin{array}{cc}10 \\
\text { Favours[Control] }\end{array}$ & 100 \\
\hline
\end{tabular}

Figure 4. Forest plots for MACE incidence for patients stratified by different clinical presentation. Forest plots were generated using Review Manager 5.2.0 for patients with stable angina, ACS or mixed disease presentation from individual and pooled trials. The incidence of MACE was expressed as a dichotomous variable, and the results were expressed as odds ratios (OR) with $95 \%$ confidence intervals (Cl). Heterogeneity across trials was evaluated with $\mathrm{I}^{2}$ statistic, defined as $\mathrm{I}^{2}>50 \%$. Because the heterogeneity was low, a fixed-effect model was used.

doi:10.1371/journal.pone.0113352.g004

\section{Analysis of the effects of high-dose statins on follow-up clinical events for subgroups of patients}

To determine the effects of high-dose statin loading on follow-up clinical events, we assessed the number of patients undergoing death, spontaneous MI, TVR or MACE events in the follow-up period after PCI. High-dose statin treatment did not significantly reduce overall mortality or spontaneous $\mathrm{MI}(\mathrm{P}=0.15$ and $\mathrm{P}=0.37$, respectively), with similar findings for subgroups stratified according to disease type or prior statin use (Table 5). TVR was significantly reduced by highdose statin pretreatment (overall $\mathrm{P}=0.02$ ), with high-dose statin pretreatment most effective for the ACS group and the prior low-dose statin group $(\mathrm{P}=0.01$ 


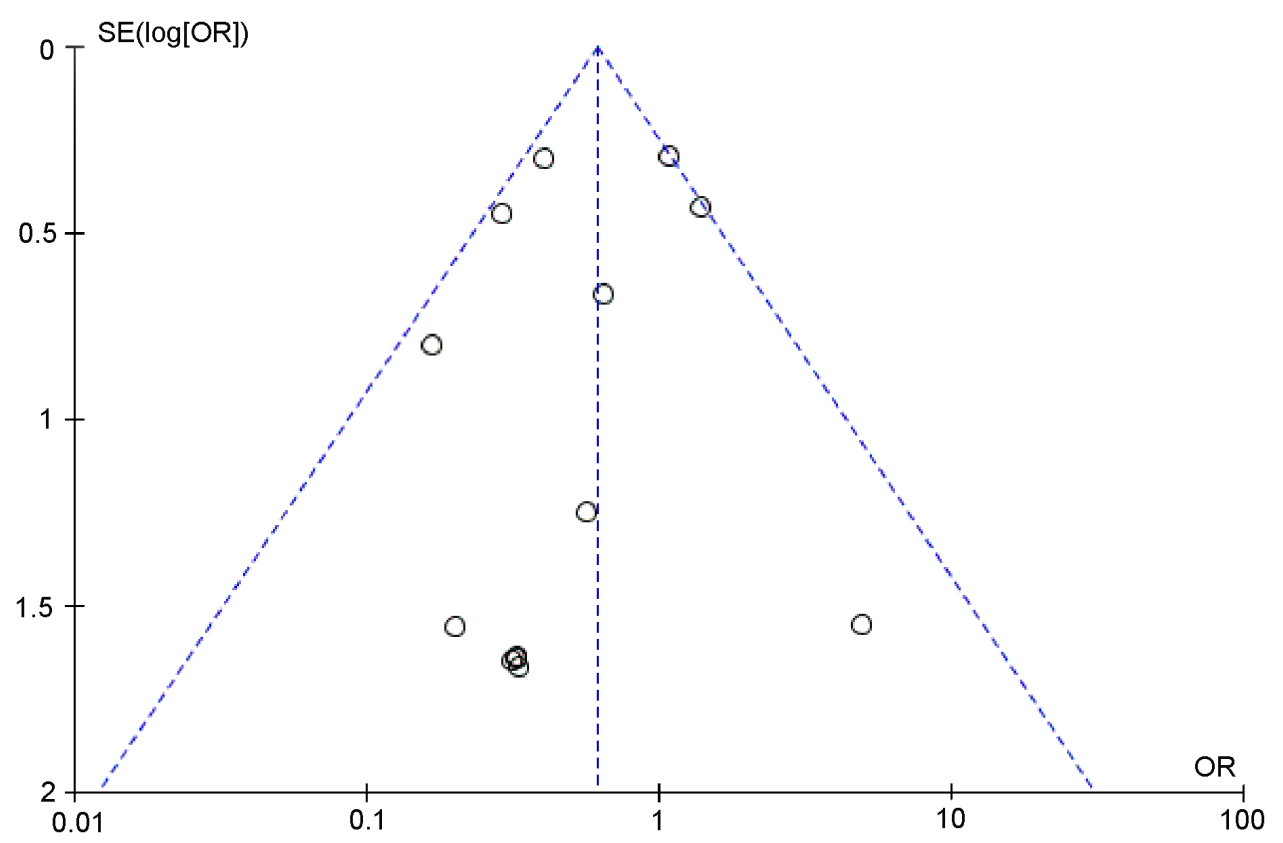

Figure 5. Funnel plot for MACE incidence to rule out publication bias. Funnel plot was generated using a fixed-effect model by Review Manager 5.2.0.

doi:10.1371/journal.pone.0113352.g005

and $\mathrm{P}=0.04$, respectively). A statistical difference in the incidence of MACE was also observed overall $(\mathrm{P}=0.002)$, for both statin-naïve $(\mathrm{P}=0.02)$ and prior lowdose statin $(\mathrm{P}=0.003)$ patients, and for patients with ACS $(\mathrm{P}=0.003)$, but not for patients with stable angina $(\mathrm{P}=0.12)$. These results provide additional support for the applicability of high-dose statins for patients with ACS, for both statin-naive and prior low-dose statin patients.

\section{Discussion}

This meta-analysis indicated that high-dose statin pretreatment can result in a significant reduction of PMI and cardiovascular events in patients undergoing elective PCI. This basic finding is consistent with previous meta-analysis studies $[14,19]$; however, our study includes more RCTs and more than 1200 additional patients and thus provides increased statistical power. When stratified according to clinical presentation, the positive effect was significant for patients with ACS, as previously suggested [19]. However, to our knowledge, our study is unique in that we provide the first meta-analysis showing that the positive effect of high-dose statin pretreatment on PMI and MACE (excluding PMI) is significant for both statin-naive patients and patients with prior low-dose statin therapy.

Previous meta-analyses show that the elevation of cardiac markers after PCI correlates with an increase of clinical events during follow-up $[\underline{4}, \underline{43}]$. There is still no consensus on the definition of PMI; however, we have elected to analyze all 


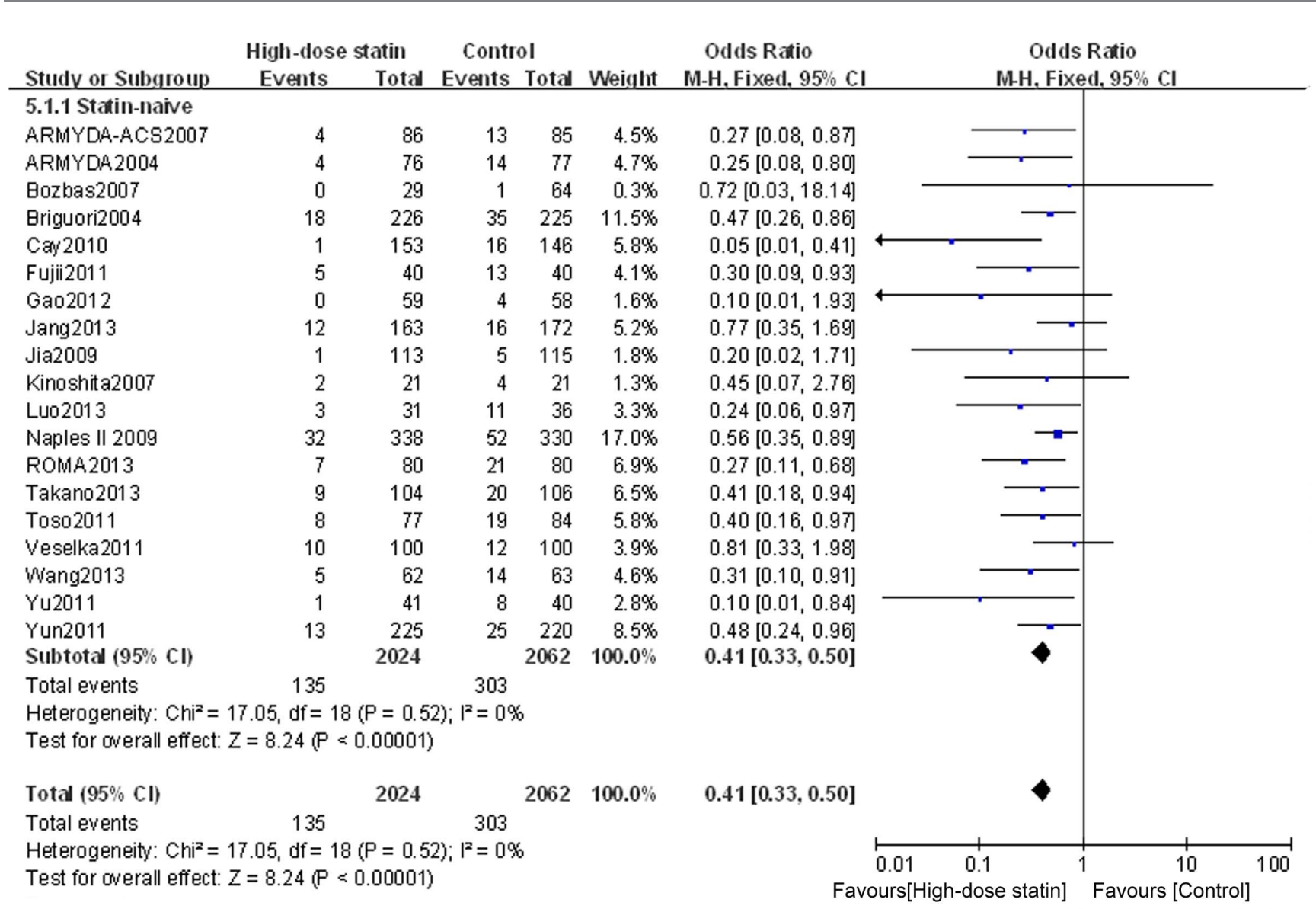

Figure 6. Funnel plot for PMI incidence for statin naïve patients. Forest plot was generated using Review Manager 5.2.0 for statin naïve patients from individual and pooled trials. The incidence of PMI was expressed as a dichotomous variable, and the results were expressed as odds ratios (OR) with $95 \%$ confidence intervals $(\mathrm{Cl})$. Heterogeneity across trials was evaluated with $\mathrm{I}^{2}$ statistic, defined as $\mathrm{I}^{2}>50 \%$. Because the heterogeneity was low, a fixed-effect model was used.

doi:10.1371/journal.pone.0113352.g006

relevant studies with quality data to provide the most comprehensive analysis possible. Despite variability in the definition of PMI, this meta-analysis demonstrated that high-dose statin pretreatment before PCI reduces the incidence of PMI. The positive effect of high-dose statin pretreatment on PMI was consistent in patients with stable and unstable coronary artery disease as well as in statin-naïve patients and prior statin-treated patients. In addition, high-dose statin pretreatment was associated with a $39 \%$ relative reduction in clinical events. The benefit in clinical events was predominantly driven by the reduction in TVR, especially for patients with ACS. The incidence of death and spontaneous MI was lower in the high-dose statin group. High-dose statin loading prior to PCI did not reduce the clinical events in patients with stable coronary artery disease. Our study confirms that reloading high-dose statin improves the clinical outcome in patients undergoing long-term therapy with statins.

Although the exact mechanisms underlying the early protective effects of high dose stains in cardiovascular events remain undetermined, the benefit of statins 


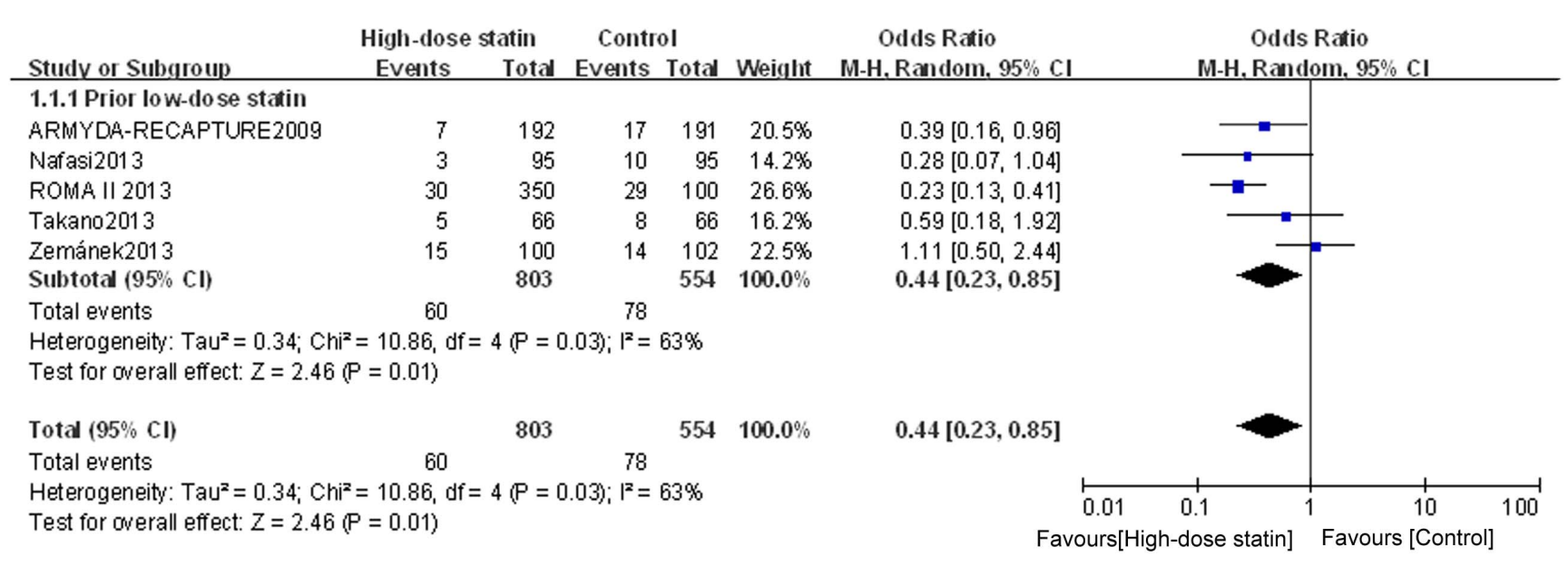

Figure 7. Funnel plot for PMI incidence for patients with prior low-dose statin use. Forest plot was generated using Review Manager 5.2.0 for patients with prior low-dose statin use from individual and pooled trials. The incidence of PMI was expressed as a dichotomous variable, and the results were expressed as odds ratios (OR) with $95 \%$ confidence intervals $(\mathrm{Cl})$. Heterogeneity across trials was evaluated with $\mathrm{I}^{2}$ statistic, defined as $\mathrm{I}^{2}>50 \%$. A randomeffect model was used for the subgroup with prior low-dose statin treatment because heterogeneity existed for this group.

doi:10.1371/journal.pone.0113352.g007

can be attributed to its pleiotropic effects, which include anti-inflammation, antiplatelet aggregation, improvement of endothelial function, and plaque stability. Periprocedural inflammatory status predicts early and late adverse outcomes after PCI [44-47]. Studies showed that a reduction of periprocedural myocardial injury after high-dose pretreatment is associated with attenuated inflammatory response $[\underline{40}, \underline{48}, \underline{49}]$. This may explain why patients with ACS may benefit most from highdose statins therapy before PCI. A clinical study [50] found that long-term treatment with statins did not result in a reduction of PMI in patients undergoing elective PCI. Animal experiments showed that the cardioprotection of statins disappears with time and that high-dose statin reloading before ischemia/ reperfusion can restore the waning protection [51]. This may suggest that highdose statin pretreatment is needed to reach the desired pleiotropic effects for patients under long-term low-dose statin treatment.

The main conclusion of this meta-analysis was similar with that from Patti et al. [14] and Alexandre et al. [19]. However, these other results should be interpreted with caution. Compared with the meta-analysis by Patti et al., we excluded the STATIN STEMI trial [52]. To our knowledge, it is the only study to evaluate the impact of high-dose statin pretreatment before primary PCI in patients with STEMI. The trial could not provide sufficient power to determine a significant difference in PMI between high-dose and low-dose statin. In their meta-analysis, Patti et al. demonstrated that high-dose statin loading before PCI significantly reduced PMI and MACE within thirty days. The clinical benefit was mainly driven by the reduction in PMI. However, when PMI was excluded from the end points, there was no significant difference in the rate of MACE within thirty days between high-dose statin and control groups $(\mathrm{OR}=0.44, \mathrm{P}=0.05)$. Our study demonstrates that high-dose statin pretreatment before PCI significantly reduces clinical events, including spontaneous MI, death, and TVR. Furthermore, we showed that high- 


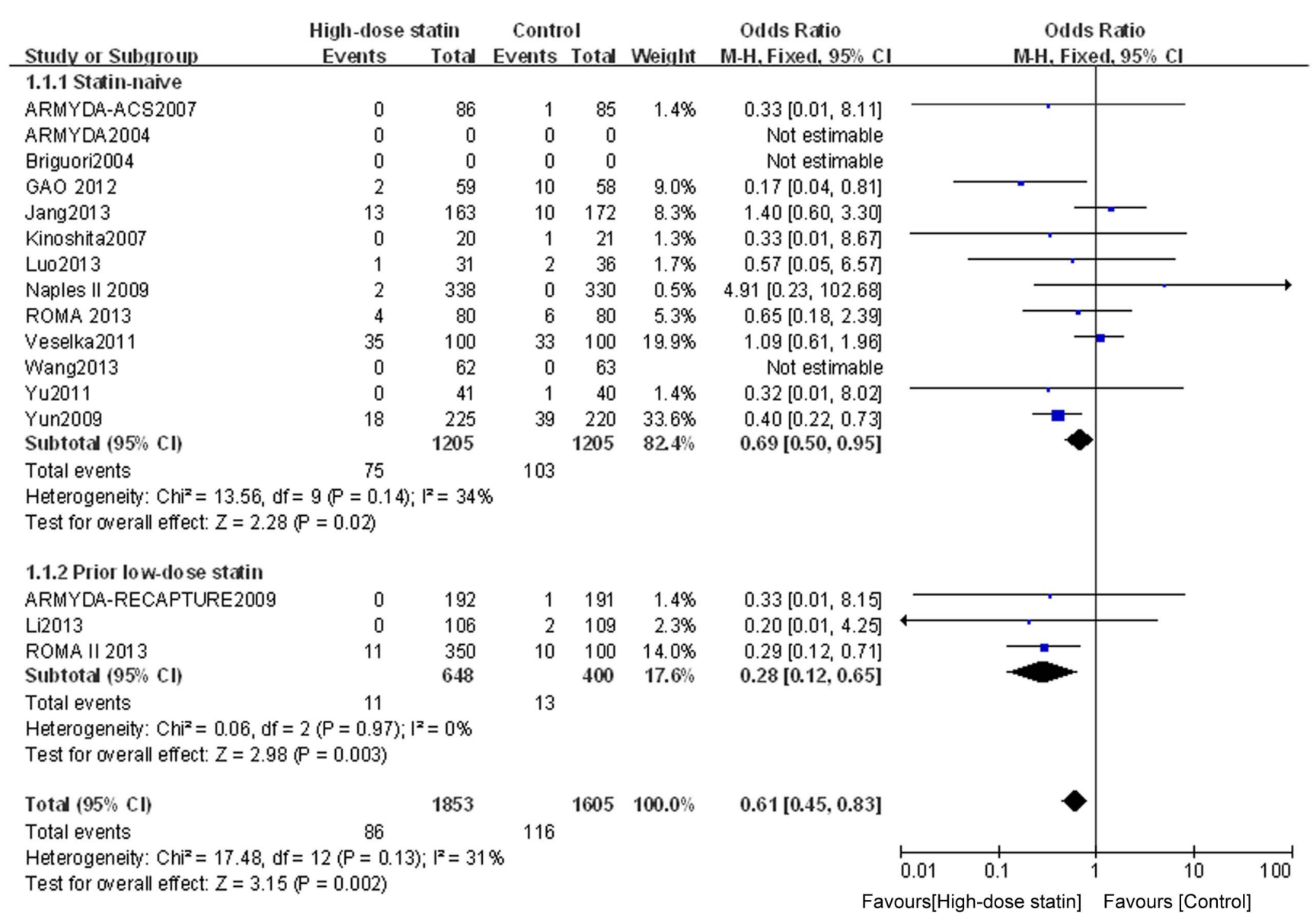

Figure 8. Forest plots for MACE incidence for patients stratified by prior low-dose statin use. Forest plots were generated using Review Manager 5.2.0 for statin naïve patients or patients with prior low-dose statin use from individual and pooled trials. The incidence of MACE was expressed as a dichotomous variable, and the results were expressed as odds ratios (OR) with $95 \%$ confidence intervals $(\mathrm{Cl})$. Heterogeneity across trials was evaluated with $\mathrm{I}^{2}$ statistic, defined as $\mathrm{I}^{2}>50 \%$. Because the heterogeneity was low, a fixed-effect model was used.

doi:10.1371/journal.pone.0113352.g008

dose statin reloading before PCI significantly reduces the occurrence of PMI and MACE during follow-up in prior low-dose statin-treated patients. Unlike the meta-analysis by Alexandre et al. [19], our study included patients under longterm low-dose statin therapy, RCTs that compared high-dose with low-dose statin treatment, and RCTs that compared different types of statin. In clinical practice, patients under long-term statin therapy constitute a large proportion of patients who undergo elective PCI. Moreover, our study included recent large scale trials $[17,24]$. Therefore, our study confirms and extends the conclusion of the metaanalysis by Alexandre et al.

The results of our meta-analysis are not consistent with the conclusions of Veselka et al. [15, 16], Jang et al. [17] and Zemanek et al. [18], which showed that high-dose statin pretreatment before PCI did not reduce the incidence of PMI and MACE in patients undergoing PCI. We think that this disagreement can be explained by different doses and durations of statins. As the optimal doses and 
Table 5. Clinical events in follow-up.

\begin{tabular}{|c|c|c|c|}
\hline Events & High-dose statin $n(\%)$ & Control n (\%) & $\mathbf{P}$ \\
\hline \multicolumn{4}{|l|}{ Stable } \\
\hline Death & $12(1.6)$ & $13(2.7)$ & 0.67 \\
\hline Spontaneous MI & $7(1.0)$ & $7(1.3)$ & 0.50 \\
\hline TVR & $31(4.2)$ & $32(6.6)$ & 0.23 \\
\hline MACE & $50(6.8)$ & $52(10.7)$ & 0.12 \\
\hline \multicolumn{4}{|l|}{ ACS } \\
\hline Death & $3(0.4)$ & $9(1.3)$ & 0.12 \\
\hline Spontaneous MI & $15(2.2)$ & $20(3.0)$ & 0.40 \\
\hline TVR & $16(2.4)$ & $34(5.0)$ & 0.01 \\
\hline MACE & $34(5.0)$ & $63(9.3)$ & 0.003 \\
\hline \multicolumn{4}{|l|}{ Mixed presentation } \\
\hline Death & $1(0.1)$ & $1(0.1)$ & 0.50 \\
\hline Spontaneous MI & $1(0.1)$ & $0(0)$ & 0.51 \\
\hline TVR & $0(0)$ & $0(0)$ & NA \\
\hline MACE & $2(0.2)$ & $1(0.1)$ & 0.67 \\
\hline \multicolumn{4}{|l|}{ Statin-naive } \\
\hline Death & $13(1.1)$ & $20(1.6)$ & 0.28 \\
\hline Spontaneous MI & $21(1.7)$ & $24(2.0)$ & 0.80 \\
\hline TVR & $41(3.4)$ & $59(4.9)$ & 0.09 \\
\hline MACE & $75(6.2)$ & $103(8.5)$ & 0.02 \\
\hline \multicolumn{4}{|l|}{ Prior low-dose statin } \\
\hline Death & $3(0.5)$ & $3(0.8)$ & 0.25 \\
\hline Spontaneous MI & $2(0.3)$ & $3(0.8)$ & 0.07 \\
\hline TVR & $6(0.9)$ & $7(1.7)$ & 0.04 \\
\hline MACE & $11(1.7)$ & $13(3.3)$ & 0.003 \\
\hline \multicolumn{4}{|l|}{ Overall } \\
\hline Death & $16(0.7)$ & $23(1.2)$ & 0.15 \\
\hline Spontaneous MI & $23(1.1)$ & $27(1.4)$ & 0.37 \\
\hline TVR & $47(2.2)$ & $66(3.5)$ & 0.02 \\
\hline MACE & $86(4.0)$ & $116(6.1)$ & 0.002 \\
\hline
\end{tabular}

Spontaneous $\mathrm{Ml}=$ spontaneous myocardial infarction; $\mathrm{ACS}=$ acute coronary syndrome;

TVR=target vessel revascularization; $\mathrm{MACE}=$ major adverse cardiovascular events;

$\mathrm{NA}=$ not available.

doi:10.1371/journal.pone.0113352.t005

duration of statins have not been determined, different therapeutic application may result in different results. Another potential explanation for the controversial outcome of the studies by Veselka et al. and Zemanek et al. is that the cardiac marker was examined at a later point in time (16 to 24 hours after PCI), potentially missing the opportunity to identify PMI. In addition, the discordant results may be attributed to a lower incidence of increased CK-MB in the highdose statin loading group, compared to the control group.

There are several limitations in this meta-analysis. First, the studies used different definitions of PMI and different treatment strategies. To date, it is not 
possible to find an ideal marker for a universally accepted definition of PMI. Different doses, durations and types of statins may have different effects on PMI and MACE. A standardized statin protocol would help to eliminate the profounder. Secondly, although many studies provided data about PMI and MACE, one out of the twenty-four studies did not report the incidence of PMI, and eight out of twenty-four studies did not provide the incidence of MACE. Finally, we did not have access to patient-level data; therefore, we did not exclude the influence of different antithrombotic strategies.

\section{Conclusions}

High-dose statin pretreatment can result in a significant reduction in PMI and cardiovascular events in patients undergoing elective PCI. The positive effects of high-dose statin pretreatment on PMI and MACE are significant for statin-naive and prior low-dose statin-treated patients. The positive effect of high-dose statin pretreatment on MACE is significant for patients with ACS. The information provided in this analysis should be useful in the selection of patients who have the greatest potential for success in the use of high-dose statin loading prior to PCI. In particular, we suggest that high-dose pretreatment should be used regardless of prior low-dose statin use for patients with ACS, but that the high-dose statin loading can be eliminated for patients with stable angina to avoid unnecessary statin exposure and unwarranted expenditure on statins.

\section{Supporting Information}

Checklist S1. PRISMA Checklist.

doi:10.1371/journal.pone.0113352.s001 (DOC)

\section{Acknowledgments}

We thank Mr. Zhizhong Gong for his kind help with statistical methods. We thank LetPub (www.letpub.com) for linguistic assistance during the preparation of this manuscript.

\section{Author Contributions}

Conceived and designed the experiments: Y. Zhou. Performed the experiments: LW PP OZ XX SY. Analyzed the data: LW PP Y. Zhao. Wrote the paper: LW.

\section{References}

1. Herrmann J (2005) Peri-procedural myocardial injury: 2005 update. Eur Heart J 26: 2493-2519.

2. Lansky AJ, Stone GW (2010) Periprocedural myocardial infarction: prevalence, prognosis, and prevention. Circ Cardiovasc Interv 3: 602-610. 
3. Park DW, Kim YH, Yun SC, Ahn JM, Lee JY, et al. (2013) Frequency, causes, predictors, and clinical significance of peri-procedural myocardial infarction following percutaneous coronary intervention. Eur Heart J 34: 1662-1669.

4. Jang JS, Jin HY, Seo JS, Yang TH, Kim DK, et al. (2013) Prognostic value of creatine kinasemyocardial band isoenzyme elevation following percutaneous coronary intervention: a meta-analysis. Catheter Cardiovasc Interv 81: 959-967.

5. Di Sciascio G, Patti G, Pasceri V, Gatto L, Colonna G, et al. (2010) Effectiveness of in-laboratory highdose clopidogrel loading versus routine pre-load in patients undergoing percutaneous coronary intervention: results of the ARMYDA-5 PRELOAD (Antiplatelet therapy for Reduction of MYocardial Damage during Angioplasty) randomized trial. J Am Coll Cardiol 56: 550-557.

6. Blankenship JC, Tasissa G, O'Shea JC, Iliadis EA, Bachour FA, et al. (2001) Effect of glycoprotein $\mathrm{Ilb} / \mathrm{lll}$ a receptor inhibition on angiographic complications during percutaneous coronary intervention in the ESPRIT trial. J Am Coll Cardiol 38: 653-658.

7. Ellis SG, Brener SJ, Lincoff AM, Moliterno DJ, Whitlow PL, et al. (2001) beta-blockers before percutaneous coronary intervention do not attenuate postprocedural creatine kinase isoenzyme rise. Circulation 104: 2685-2688.

8. Park H, Otani H, Noda T, Sato D, Okazaki T, et al. (2013) Intracoronary followed by intravenous administration of the short-acting beta-blocker landiolol prevents myocardial injury in the face of elective percutaneous coronary intervention. Int J Cardiol 167: 1547-1551.

9. Macin SM, Perna ER, Farias EF, Franciosi V, Cialzeta JR, et al. (2005) Atorvastatin has an important acute anti-inflammatory effect in patients with acute coronary syndrome: results of a randomized, double-blind, placebo-controlled study. Am Heart J 149: 451-457.

10. Sanguigni V, Pignatelli P, Lenti L, Ferro D, Bellia A, et al. (2005) Short-term treatment with atorvastatin reduces platelet CD40 ligand and thrombin generation in hypercholesterolemic patients. Circulation 111: 412-419.

11. Wagner AH, Kohler T, Ruckschloss U, Just I, Hecker M (2000) Improvement of nitric oxide-dependent vasodilatation by HMG-CoA reductase inhibitors through attenuation of endothelial superoxide anion formation. Arterioscler Thromb Vasc Biol 20: 61-69.

12. Califf RM, Abdelmeguid AE, Kuntz RE, Popma JJ, Davidson CJ, et al. (1998) Myonecrosis after revascularization procedures. J Am Coll Cardiol 31: 241-251.

13. Crisby M, Nordin-Fredriksson G, Shah PK, Yano J, Zhu J, et al. (2001) Pravastatin treatment increases collagen content and decreases lipid content, inflammation, metalloproteinases, and cell death in human carotid plaques: implications for plaque stabilization. Circulation 103: 926-933.

14. Patti G, Cannon CP, Murphy SA, Mega S, Pasceri V, et al. (2011) Clinical benefit of statin pretreatment in patients undergoing percutaneous coronary intervention: a collaborative patient-level meta-analysis of 13 randomized studies. Circulation 123: 1622-1632.

15. Veselka J, Zemanek D, Hajek P, Maly M, Adlova R, et al. (2009) Effect of two-day atorvastatin pretreatment on the incidence of periprocedural myocardial infarction following elective percutaneous coronary intervention: a single-center, prospective, and randomized study. Am J Cardiol 104: 630-633.

16. Veselka J, Zemanek D, Hajek P, Maly M, Adlova R, et al. (2011) Effect of two-day atorvastatin pretreatment on long-term outcome of patients with stable angina pectoris undergoing elective percutaneous coronary intervention. Am J Cardiol 107: 1295-1299.

17. Jang Y, Zhu J, Ge J, Kim YJ, Ji C, et al. (2013) Preloading with atorvastatin before percutaneous coronary intervention in statin-naive Asian patients with non-ST elevation acute coronary syndromes: A randomized study. J Cardiol 63: 335-343.

18. Zemanek D, Branny M, Martinkovicova L, Hajek P, Maly M, et al. (2013) Effect of seven-day atorvastatin pretreatment on the incidence of periprocedural myocardial infarction following percutaneous coronary intervention in patients receiving long-term statin therapy. A randomized study. Int J Cardiol 168: 2494-2497.

19. Benjo AM, El-Hayek GE, Messerli F, Dinicolantonio JJ, Hong MK, et al. (2013) High dose statin loading prior to percutaneous coronary intervention decreases cardiovascular events: A meta-analysis of randomized controlled trials. Catheter Cardiovasc Interv.

20. Higgins JP, Altman DG, Gotzsche PC, Juni P, Moher D, et al. (2011) The Cochrane Collaboration's tool for assessing risk of bias in randomised trials. BMJ 343: d5928. 
21. Jadad AR, Moore RA, Carroll D, Jenkinson C, Reynolds DJ, et al. (1996) Assessing the quality of reports of randomized clinical trials: is blinding necessary? Control Clin Trials 17: 1-12.

22. Li Q, Deng SB, Xia S, Du JL, She Q (2013) Impact of intensive statin use on the level of inflammation and platelet activation in stable angina after percutaneous coronary intervention: a clinical study. Med Clin (Barc) 140: 532-536.

23. Di Sciascio G, Patti G, Pasceri V, Gaspardone A, Colonna G, et al. (2009) Efficacy of atorvastatin reload in patients on chronic statin therapy undergoing percutaneous coronary intervention: results of the ARMYDA-RECAPTURE (Atorvastatin for Reduction of Myocardial Damage During Angioplasty) Randomized Trial. J Am Coll Cardiol 54: 558-565.

24. Sardella G, Lucisano L, Mancone M, Conti G, Calcagno S, et al. (2013) Comparison of high reloading ROsuvastatin and Atorvastatin pretreatment in patients undergoing elective $\mathrm{PCl}$ to reduce the incidence of MyocArdial periprocedural necrosis. The ROMA II trial. Int J Cardiol 168: 3715-3720.

25. Nafasi L, Rahmani R, Shafiee A, Salari A, Abdollahi A, et al. (2014) Can a high reloading dose of atorvastatin prior to percutaneous coronary intervention reduce periprocedural myocardial infarction? Curr Med Res Opin 30: 381-386.

26. Pasceri V, Patti G, Nusca A, Pristipino C, Richichi G, et al. (2004) Randomized trial of atorvastatin for reduction of myocardial damage during coronary intervention: results from the ARMYDA (Atorvastatin for Reduction of MYocardial Damage during Angioplasty) study. Circulation 110: 674-678.

27. Briguori C, Colombo A, Airoldi F, Violante A, Focaccio A, et al. (2004) Statin administration before percutaneous coronary intervention: impact on periprocedural myocardial infarction. Eur Heart J 25: $1822-1828$.

28. Patti G, Pasceri V, Colonna G, Miglionico M, Fischetti D, et al. (2007) Atorvastatin pretreatment improves outcomes in patients with acute coronary syndromes undergoing early percutaneous coronary intervention: results of the ARMYDA-ACS randomized trial. J Am Coll Cardiol 49: 1272-1278.

29. Bozbas H, Yildirir A, Mermer S, Konas D, Atar I, et al. (2007) Does pravastatin therapy affect cardiac enzyme levels after percutaneous coronary intervention? Adv Ther 24: 493-504.

30. Kinoshita M, Matsumura S, Sueyoshi K, Ogawa S, Fukuda K (2007) Randomized trial of statin administration for myocardial injury: is intensive lipid-lowering more beneficial than moderate lipidlowering before percutaneous coronary intervention? Circ J 71: 1225-1228.

31. Briguori C, Visconti G, Focaccio A, Golia B, Chieffo A, et al. (2009) Novel approaches for preventing or limiting events (Naples) II trial: impact of a single high loading dose of atorvastatin on periprocedural myocardial infarction. J Am Coll Cardiol 54: 2157-2163.

32. Jia XW, Fu XH, Zhang J, Gu XS, Fan WZ, et al. (2009) Intensive cholesterol lowering with statin improves the outcomes of percutaneous coronary intervention in patients with acute coronary syndrome. Chin Med J (Engl) 122: 659-664.

33. Cay S, Cagirci G, Sen N, Balbay Y, Durmaz T, et al. (2010) Prevention of peri-procedural myocardial injury using a single high loading dose of rosuvastatin. Cardiovasc Drugs Ther 24: 41-47.

34. Toso A, Leoncini M, Maioli M, Gallopin M, Tedeschi D, et al. (2011) Short-term high-dose atorvastatin for periprocedural myocardial infarction prevention in patients with renal dysfunction. $\mathrm{J}$ Cardiovasc Med (Hagerstown) 12: 318-321.

35. Yun KH, Oh SK, Rhee SJ, Yoo NJ, Kim NH, et al. (2011) 12-month follow-up results of high dose rosuvastatin loading before percutaneous coronary intervention in patients with acute coronary syndrome. Int J Cardiol 146: 68-72.

36. Fujii K, Kawasaki D, Oka K, Akahori H, Iwasaku T, et al. (2011) The impact of pravastatin pretreatment on periprocedural microcirculatory damage in patients undergoing percutaneous coronary intervention. JACC Cardiovasc Interv 4: 513-520.

37. Yu XL, Zhang HJ, Ren SD, Geng J, Wu TT, et al. (2011) Effects of loading dose of atorvastatin before percutaneous coronary intervention on periprocedural myocardial injury. Coron Artery Dis 22: 87-91.

38. Gao Y, Jia ZM, Sun YJ, Zhang ZH, Ren LN, et al. (2012) Effect of high-dose rosuvastatin loading before percutaneous coronary intervention in female patients with non-ST-segment elevation acute coronary syndrome. Chin Med J (Engl) 125: 2250-2254. 
39. Sardella G, Conti G, Donahue M, Mancone M, Canali E, et al. (2013) Rosuvastatin pretreatment in patients undergoing elective $\mathrm{PCI}$ to reduce the incidence of myocardial periprocedural necrosis: the ROMA trial. Catheter Cardiovasc Interv 81: E36-43.

40. Luo J, Li J, Shen X, Hu X, Fang Z, et al. (2013) The effects and mechanisms of high loading dose rosuvastatin therapy before percutaneous coronary intervention in patients with acute coronary syndrome. Int J Cardiol 167: 2350-2353.

41. Wang Z, Dai H, Xing M, Yu Z, Lin X, et al. (2013) Effect of a single high loading dose of rosuvastatin on percutaneous coronary intervention for acute coronary syndromes. J Cardiovasc Pharmacol Ther 18: 327-333.

42. Takano H, Ohba T, Yamamoto E, Miyachi H, Inui K, et al. (2013) Usefulness of rosuvastatin to prevent periprocedural myocardial injury in patients undergoing elective coronary intervention. Am J Cardiol 111: 1688-1693.

43. Feldman DN, Kim L, Rene AG, Minutello RM, Bergman G, et al. (2011) Prognostic value of cardiac troponin-I or troponin-T elevation following nonemergent percutaneous coronary intervention: a metaanalysis. Catheter Cardiovasc Interv 77: 1020-1030.

44. Buffon A, Liuzzo G, Biasucci LM, Pasqualetti P, Ramazzotti V, et al. (1999) Preprocedural serum levels of C-reactive protein predict early complications and late restenosis after coronary angioplasty. J Am Coll Cardiol 34: 1512-1521.

45. Walter DH, Fichtlscherer S, Sellwig M, Auch-Schwelk W, Schachinger V, et al. (2001) Preprocedural C-reactive protein levels and cardiovascular events after coronary stent implantation. J Am Coll Cardiol 37: 839-846.

46. Delhaye C, Maluenda G, Wakabayashi K, Ben-Dor I, Lemesle G, et al. (2010) Long-term prognostic value of preprocedural C-reactive protein after drug-eluting stent implantation. Am J Cardiol 105: 826832.

47. Delhaye C, Sudre A, Lemesle G, Marechaux S, Broucqsault D, et al. (2009) Preprocedural highsensitivity C-reactive protein predicts death or myocardial infarction but not target vessel revascularization or stent thrombosis after percutaneous coronary intervention. Cardiovasc Revasc Med 10: $144-150$.

48. Patti G, Chello M, Pasceri V, Colonna D, Nusca A, et al. (2006) Protection from procedural myocardial injury by atorvastatin is associated with lower levels of adhesion molecules after percutaneous coronary intervention: results from the ARMYDA-CAMs (Atorvastatin for Reduction of MYocardial Damage during Angioplasty-Cell Adhesion Molecules) substudy. J Am Coll Cardiol 48: 1560-1566.

49. Patti G, Chello M, Gatto L, Alfano G, Miglionico M, et al. (2010) Short-term atorvastatin preload reduces levels of adhesion molecules in patients with acute coronary syndrome undergoing percutaneous coronary intervention. Results from the ARMYDA-ACS CAMs (Atorvastatin for Reduction of MYocardial Damage during Angioplasty-Cell Adhesion Molecules) substudy. J Cardiovasc Med (Hagerstown) 11: 795-800.

50. Gordin J, Haider A, Swaminathan RV, Kim LK, Minutello RM, et al. (2012) Impact of long-term statin therapy on postprocedural myocardial infarction in patients undergoing nonemergency percutaneous coronary intervention. Am J Cardiol 110: 1397-1404.

51. Mensah K, Mocanu MM, Yellon DM (2005) Failure to protect the myocardium against ischemia/ reperfusion injury after chronic atorvastatin treatment is recaptured by acute atorvastatin treatment: a potential role for phosphatase and tensin homolog deleted on chromosome ten? J Am Coll Cardiol 45: $1287-1291$.

52. Kim JS, Kim J, Choi D, Lee CJ, Lee SH, et al. (2010) Efficacy of high-dose atorvastatin loading before primary percutaneous coronary intervention in ST-segment elevation myocardial infarction: the STATIN STEMI trial. JACC Cardiovasc Interv 3: 332-339. 